- Basics of Cosmology

- Friedmann equations

- Thermal history

- Conceptual problems of classical cosmology

- Hot Big Bang

- Cosmic Microwave Background

- Big Bang Nucleosynthesis

- Baryogenesis

- Phase trnasitions and topological defects

- Missing mass: evidence for new physics

- Dark matter problem

- Dark energy problem

- Particle physics solutions

- Inflationary cosmology

- How it works and what it does

- Creation of seeds for structure

- Comparison with observations 


\section{Astronomical Distance Scales}
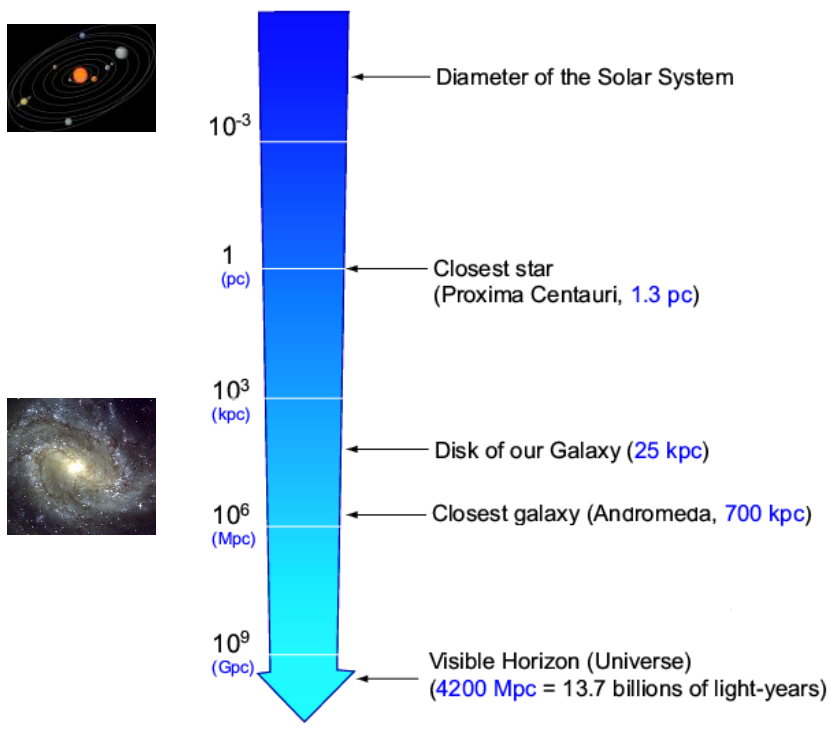

Parsecs (pc)

1 parsec $=3.26$ light-years $\sim 3 \times 10^{13} \mathrm{~km}$ 
Astronomical Distance Scales

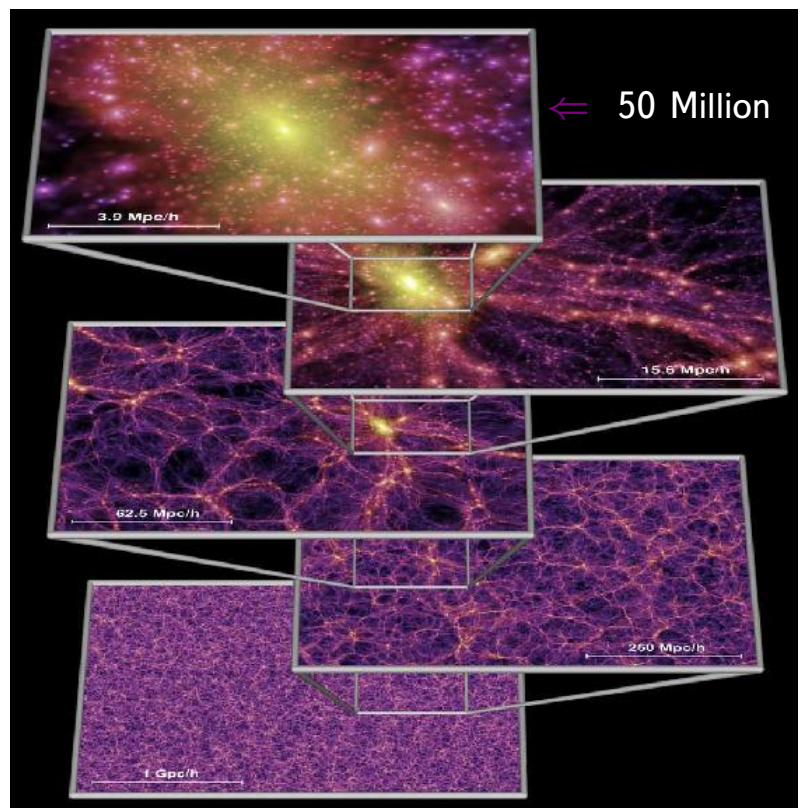

light years 


\section{Looking Back in Time}

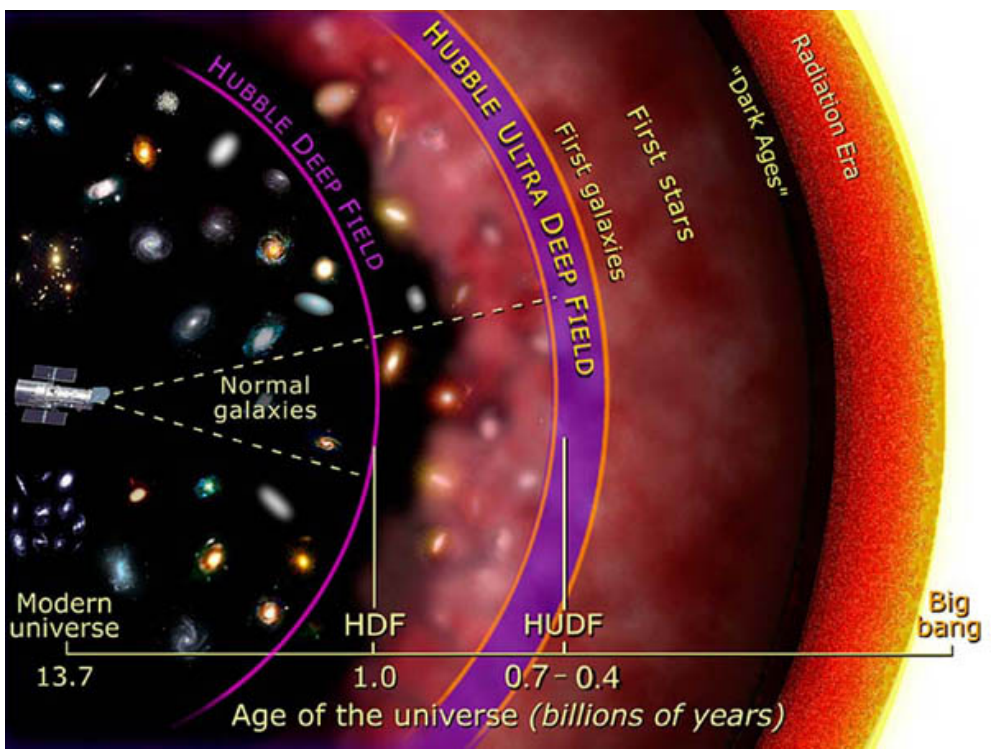




\section{Space and time}

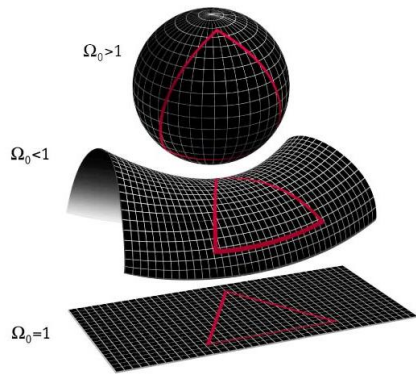

- Space is three dimensional and all points are alike. There are three possible realizations of a homogeneous isotropic space.

- Space and time make dynamical framework 


\section{Dynamical Frameworks}

Einstein equations

$$
R_{\mu \nu}-\frac{1}{2} g_{\mu \nu} R=8 \pi G T_{\mu \nu}
$$

Crucial assumption: The Universe is homogeneous.

Friedmann solution: The Universe should expand.

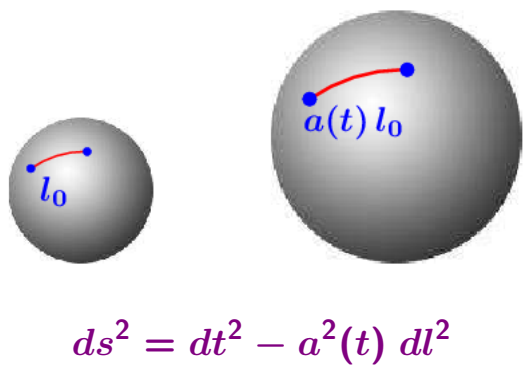




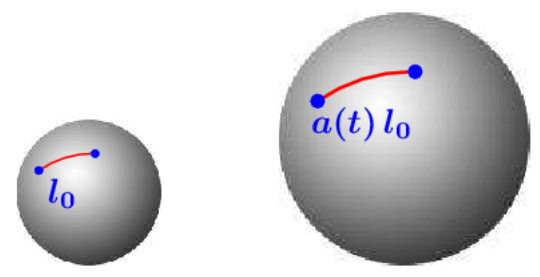

$$
d s^{2}=d t^{2}-a^{2}(t) d l^{2}
$$

$$
d l^{2}=\frac{d r^{2}}{1-k r^{2}}+r^{2}\left(d \theta^{2}+\sin ^{2} \theta d \phi^{2}\right)
$$

${ }^{(3)} R=\frac{6 k}{a^{2}(t)} \quad \begin{cases}k=-1 & \text { Open } \\ k=0 & \text { Flat } \\ k=+1 & \text { Closed }\end{cases}$ 
Assume ideal fluid for the energy momentum tensor

$$
T_{\mu \nu}=\left(\begin{array}{llll}
\rho & 0 & 0 & 0 \\
0 & p & 0 & 0 \\
0 & 0 & p & 0 \\
0 & 0 & 0 & p
\end{array}\right)
$$

Einstein equations written for homogeneous isotropic world give

$$
\begin{gathered}
\left(\frac{\dot{a}}{a}\right)^{2}=\frac{8 \pi G}{3} \rho-\frac{k}{a^{2}} \\
\frac{\ddot{a}}{a}=-\frac{4 \pi G}{3}(\rho+3 p)
\end{gathered}
$$




\section{Friedmann equations: the physics behind}

Friedmann equation

$$
\left(\frac{\dot{a}}{a}\right)^{2}=\frac{8 \pi G}{3} \rho-\frac{k}{a^{2}}
$$

has familiar Newtonian counterpart. Indeed

$$
\frac{1}{2} \dot{a}^{2}-\frac{4 \pi G}{3} \rho a^{2}=-\frac{k}{2}
$$

With $r=a r_{0}$ and $M=\frac{4 \pi}{3} \rho r^{3}$ Friedmann equation takes the form of energy conservation for a test particles bounded in the gravitaional potential

$$
\frac{1}{2} \dot{r}^{2}-\frac{G M}{r}=-\frac{k r_{0}^{2}}{2}
$$




\section{Geometry versus Universe future}

$$
\frac{1}{2} \dot{r}^{2}-\frac{G M}{r}=-\frac{k r_{0}^{2}}{2}
$$

$k=+1 \quad$ Binding energy negative Universe will recollapce $k=-1 \quad$ Binding energy positive Universe will expand forever $k=0 \quad$ Critical density $\quad \rho_{c} \equiv \frac{3}{8 \pi G}\left(\frac{\dot{a}}{a}\right)^{2}$

$$
\left(\frac{\dot{a}}{a}\right)^{2}=\frac{8 \pi G}{3} \rho-\frac{k}{a^{2}}
$$


To find critical density $\rho_{c} \equiv \frac{3}{8 \pi G}\left(\frac{\dot{a}}{a}\right)^{2}$ one needs to know

$$
H \equiv \frac{\dot{a}}{a} \quad \text { Hubble "constant" }
$$

Using $r(t)=a(t) r_{0}$ one can find the velocity with which distance between two points increases

$$
v \equiv \dot{a} r_{0}=\frac{\dot{a}}{a} a r_{0}=H r
$$

This gives the Hubble law

$$
v=H r
$$

Horizon: $\quad v \sim 1 \quad$ at $\quad r \sim H^{-1}$

But at $r \ll H^{-1} \quad$ Newtonian mechanics should be valid 


\section{Expansion of the Universe}

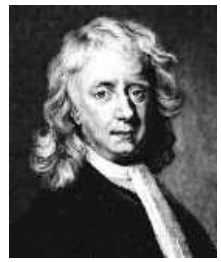

- Newton did not know that one should worry about horizons, but he worried why the Universe does not collapse under the pull of gravity.

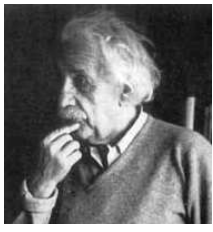

- In 1917 Einstein added cosmological constant to his equations thinking it will provide static solutions.

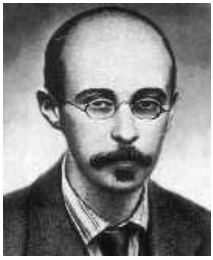

- In 1922 Friedmann had shown that the Universe must expand as a whole. After some debate Einstein admitted mistake and called the introduction of a cosmological constant "the greatest blunder of my life". 


\section{Why the Universe did not collapsed under the pull of gravity?}

Resolution is in awkward initial conditions called Big Bang

$$
\frac{1}{2} \dot{r}^{2}-\frac{G M}{r}=-\frac{k r_{0}^{2}}{2}
$$

which imply enormous fine-tuning and which are hard to accept.

We will see how the modern inflationary cosmology solves this problem of initial conditions. 


\section{Cosmological Parameters}

Introduced to parametize Fiedmann equation and its solution $a(t)$

\begin{tabular}{ll}
\hline$t$ & Age \\
$H=\dot{a} / a$ & Hubble “constant" at time t \\
$\rho_{c}=3 H^{2} / 8 \pi G$ & Critical density \\
$\Omega=\rho / \rho_{c}$ & $\Omega_{B}=\rho_{B} / \rho_{c}, \quad \Omega_{r}=\rho_{r} / \rho_{c}, \ldots$ \\
$q=-\ddot{a} a / \dot{a}^{2}$ & Decceleration parameter \\
\hline
\end{tabular}

Present values $t_{0}, H_{0}, \ldots$ are called cosmological parameters $t_{0}=(13.7 \pm 0.2) \mathrm{Gyr}$ $H_{0} \equiv 100 h \mathrm{~km} \mathrm{~s}^{-1} \mathrm{Mpc}^{-1}$, where $h=0.71 \pm 0.04$ $\rho_{c}=10 h^{2} \mathrm{GeV} \mathrm{m}^{-3}$

$\Omega_{0}=1.02 \pm 0.02$ $q_{0}=-0.6 \pm 0.3$ 


\section{Matter content in the Universe}

- Light. (Relativistic degrees of freedom)

Major energy fraction at early times.

- Baryonic matter. (Stars)

Observable world today.

- Dark matter. (Should be there)

Major matter fraction today.

- Dark energy. (Vacuum)

Major energy fraction today. 
Definition: $\quad w \equiv \frac{p}{\rho}$

If $w=$ const, conservation of energy gives $\rho=a^{-3(1+w)} \rho_{0}$

Ordinary forms of matter

- Radiation ................... w $w=\frac{1}{3} \ldots \ldots . \rho=a^{-4} \rho_{0}$

- Matter ....................... $w=0$...... $\rho=a^{-3} \rho_{0}$

Hypothetical matter

- Cosmic strings ............w w $=-\frac{1}{3} \ldots . . \rho=a^{-2} \rho_{0}$

- Domain walls ............. w $w=-\frac{2}{3} \ldots . . \rho=a^{-1} \rho_{0}$

- Cosmological constant ... w $=-1 \ldots . \rho=$ const 
Friedmann equation in spatially flat Universe

$$
\left(\frac{\dot{a}}{a}\right)^{2}=\frac{8 \pi G}{3} \rho
$$

for $w=$ const gives

$$
a=\left(\frac{t}{t_{0}}\right)^{\frac{2}{3(1+w)}}
$$

In particular
- Radiation ..... w $=\frac{1}{3} \ldots . . a=\left(t / t_{0}\right)^{1 / 2}$
- Matter ........ $w=0 \ldots . . a=\left(t / t_{0}\right)^{2 / 3}$ 
In the past the Universe was dense and therefore hot 
One of the two Friedmann equations can be excluded in favour of

$$
\frac{d \rho}{d t}+3 \frac{\dot{a}}{a}(\rho+p)=0
$$

which is nothing but energy-momentum conseravtion $T_{; \nu}^{\mu \nu}=0$

And this is nothing but the First Law of thermodynamics

$$
d E+p d V=T d S
$$

Here $E=\rho V=\rho a^{3}$ is energy and $S$ is entropy. 
Friedmann expansion driven by an ideal fluid is isentropic, $d S=0$. Dissipation is negligible usually.

Entropy:

$$
\begin{aligned}
S & =\frac{2 \pi^{2}}{45} g_{*} T^{3} a^{3}=\text { const, } \\
g_{*} & =\sum_{i=\text { bosons }} g_{i}+\frac{7}{8} \sum_{j=\text { fermions }} g_{j} .
\end{aligned}
$$

Useful relation

$$
a \propto \frac{1}{T}
$$




\section{Relativistic degrees of freedom}

Particles with $\quad m \ll T$ should be counted only, i. e. $g_{*}$ is a function of temperature

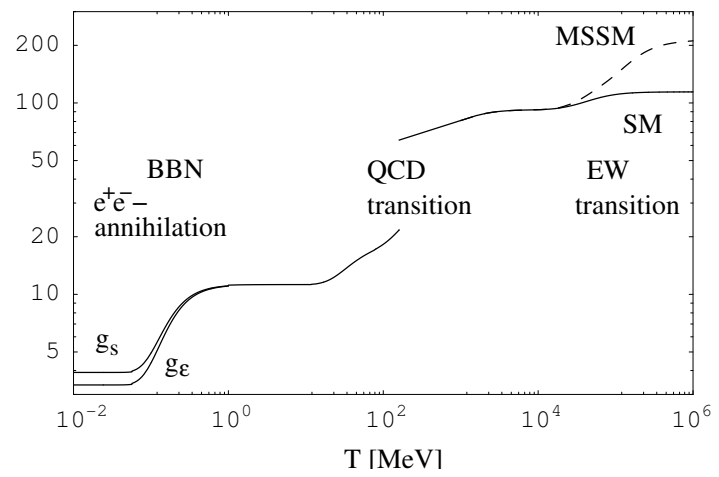




\section{Georgy Antonovich Gamov}

Nuclear physicist and cosmologist

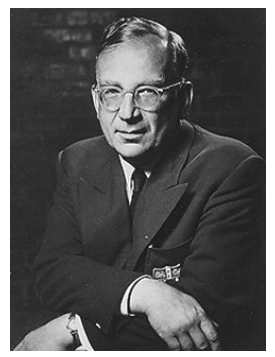

- Explained nuclear alpha decay by quantum mechanical tunneling (1928).

- His model of atomic nuclei (1929) served as the basis for the modern theories of nuclear fission and fusion.

- He developed, with Edward Teller, a theory of nuclear beta decay (1936). 


\section{Georgy Antonovich Gamov}

Nuclear physicist and cosmologist
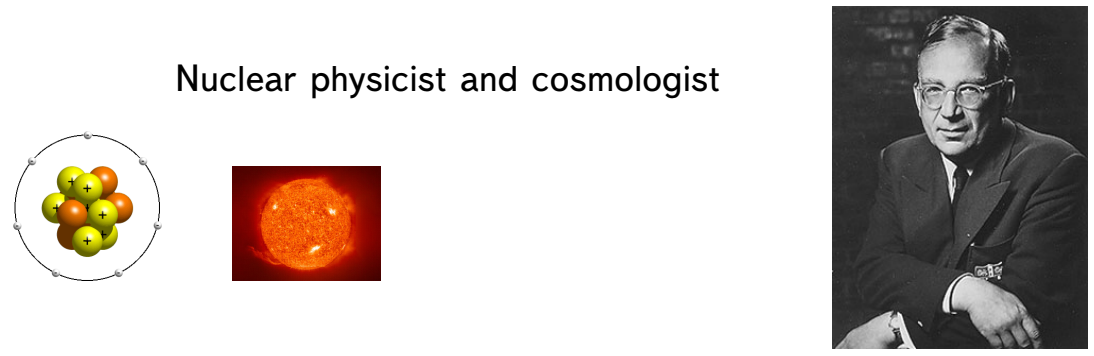

- Later he used his knowledge of nuclear reactions to interpret stellar evolution and element formation in stars (1938).

- Modeled red giants, supernovae, and neutron stars (1939). 


\section{Georgy Antonovich Gamov}

Nuclear physicist and cosmologist
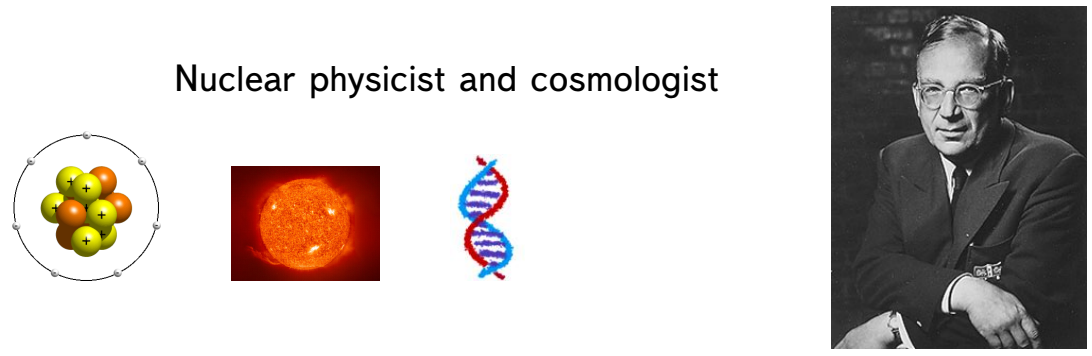

- In 1954 he proposed the concept of a genetic code and first suggested how the genetic code might be transcribed... 


\section{Georgy Antonovich Gamov}

Nuclear physicist and cosmologist
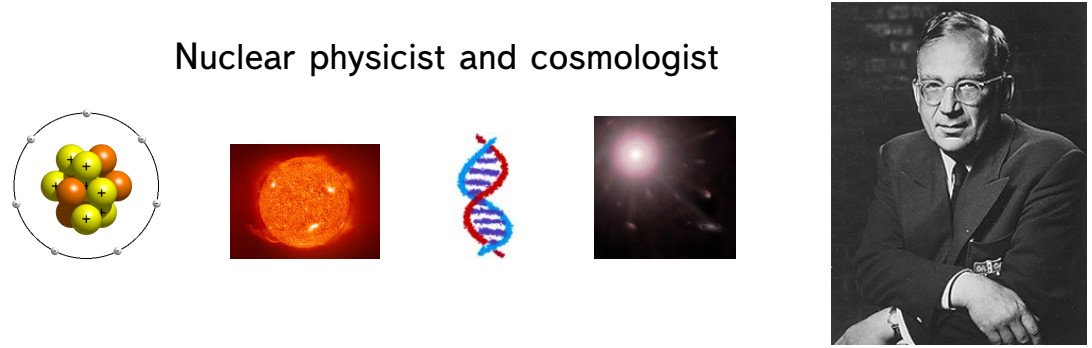

- In 1946 G. Gamov realized that ${ }^{4} \mathrm{He}$ could not have been produced in stars. He suggested, as a way out, that the early Universe itself was the Oven in which light elements were cooked up.

- He also calculated the left-over heat which should be measured today as $5^{\circ} \mathrm{K}$ CMBR.

The Hot Big Bang theory was born. 


\section{Cosmic Microwave Background Radiation}

Predicted by G. Gamov in 1946: $5 \boldsymbol{K}^{\circ}$

Measured by A. Penzias and R. Wilson in 1965: $3.5 \mathrm{~K}^{\circ}$

- $2.725 \mathrm{~K}^{\circ}$ above absolute zero

- $\mathrm{mm}-\mathrm{cm}$ wavelength

- 410 photons per cubic centimeter

- 10 trillion photons per second per squared centimeter

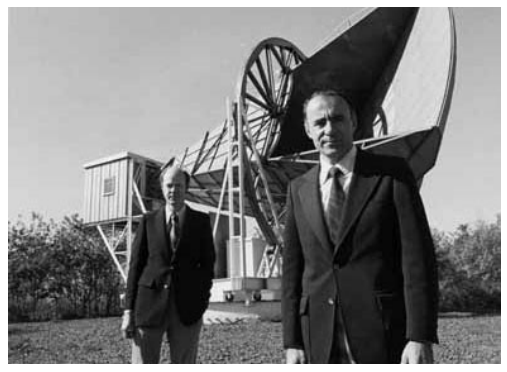




\section{Cosmic Microwave Background Radiation}

Predicted by G. Gamov in 1946: $5 \boldsymbol{K}^{\circ}$

Measured by A. Penzias and R. Wilson in 1965: $3.5 K^{\circ}$

- $2.725 \mathrm{~K}^{\circ}$ above absolute zero

- $\mathrm{mm}-\mathrm{cm}$ wavelength

- 410 photons per cubic centimeter

- 10 trillion photons per second per squared centimeter

- Few percent of TV "snow"

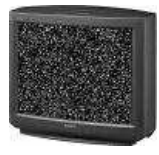




\section{Perfect Blackbody}

The CMBR spectrum is strictly blackbody, $T=2.725 \mathrm{~K}$

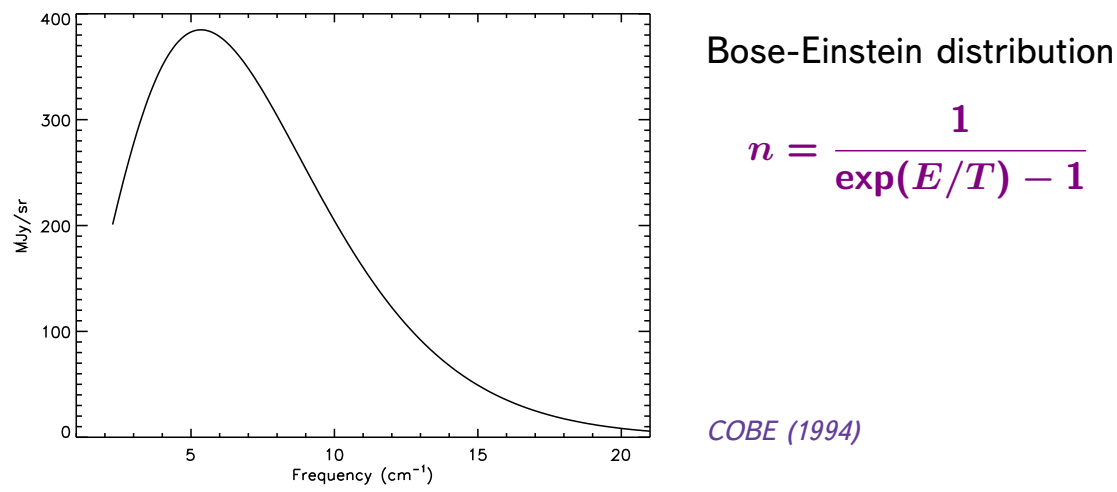

There is no explanation to it but the hot Big Bang 
Matter is ionized at temperatures higher than hydrogen ionization energy $\quad E_{\text {ion }}=13.6 \mathrm{eV}$. At lower $T$ neutral atoms start to form.

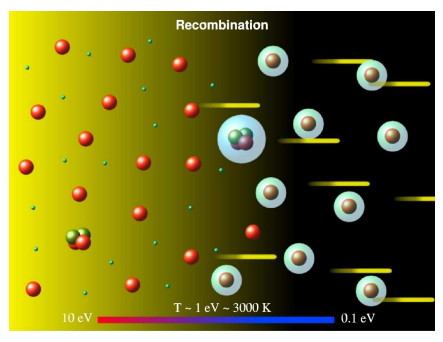

$$
\frac{n_{e} n_{p}}{n_{H}}=\left(\frac{m_{e} T}{2 \pi}\right)^{3 / 2} \mathrm{e}^{-E_{\text {ion }} / T}
$$

Univerese became transparent for radiation when

$$
\sigma_{\gamma e} n_{e} \sim t
$$

Here $\sigma_{\gamma e}=8 \pi \alpha^{2} / 3 m_{e}^{2}$ is the Compton cross-section.

Numerically $T_{\text {ls }}=0.26 \mathrm{eV}$ 


\section{Last scattering of light}

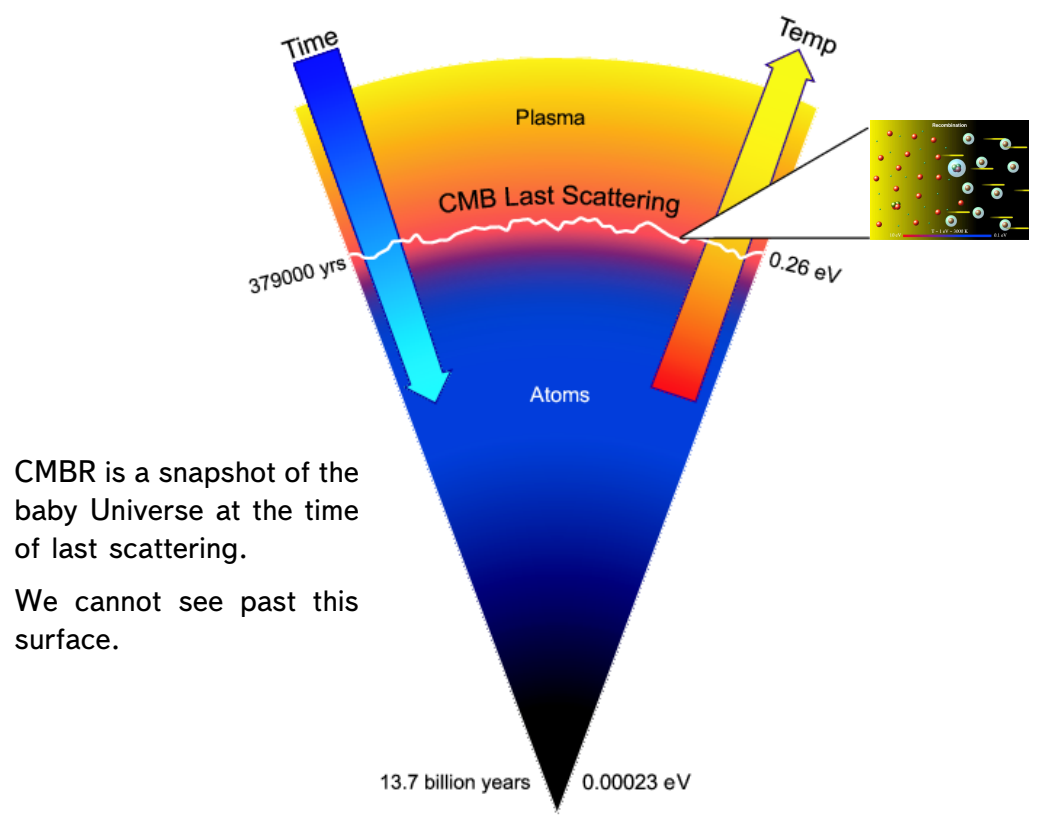




\section{Temperature map of the sky}

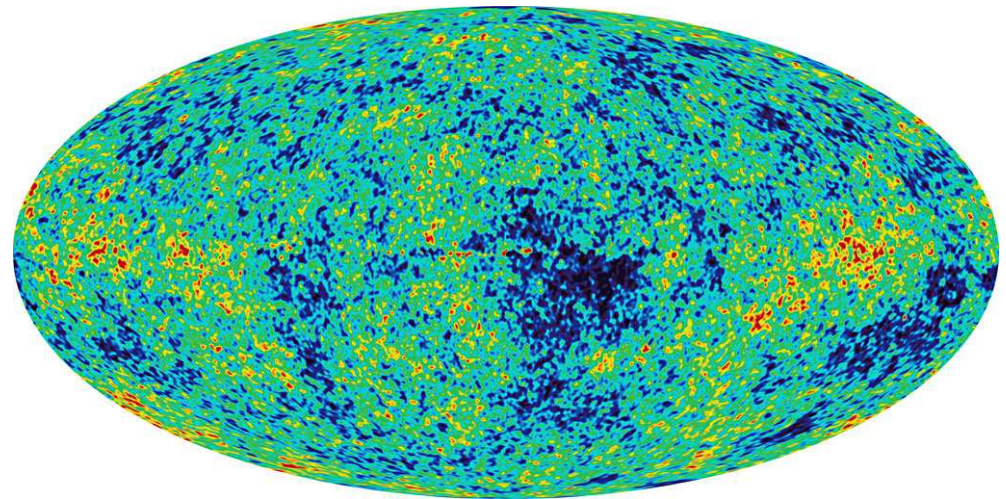

- Temperature slightly different in different patches of the sky - 1 part in 100,000. 
The temperature anisotropy, $T(\mathrm{n})$, is expanded in a spherical harmonics

$$
T(\mathrm{n})=\sum_{l, m} a_{l m} Y_{l m}(\mathrm{n}) .
$$

The angular power spectrum, $C_{l}$, is defined as

$$
C_{l}^{s k y}=\frac{1}{2 l+1} \sum_{m}\left|a_{l m}\right|^{2} .
$$

Assuming random phases, the temperature anisotropy for each multipole moment, $\Delta T_{l}$, can be associated with the angular spectrum

$$
\Delta T_{l}=\sqrt{C_{l}^{s k y} l(l+1) / 2 \pi} .
$$

The correlation function is

$$
C(\theta)=\frac{1}{4 \pi} \sum_{l}(2 l+1) C_{l} P_{l}(\cos \theta)
$$

where $P_{l}$ is the Legendre polynomial or order $l$. 


\section{CMB power spectrum: tool of Precision Cosmology}

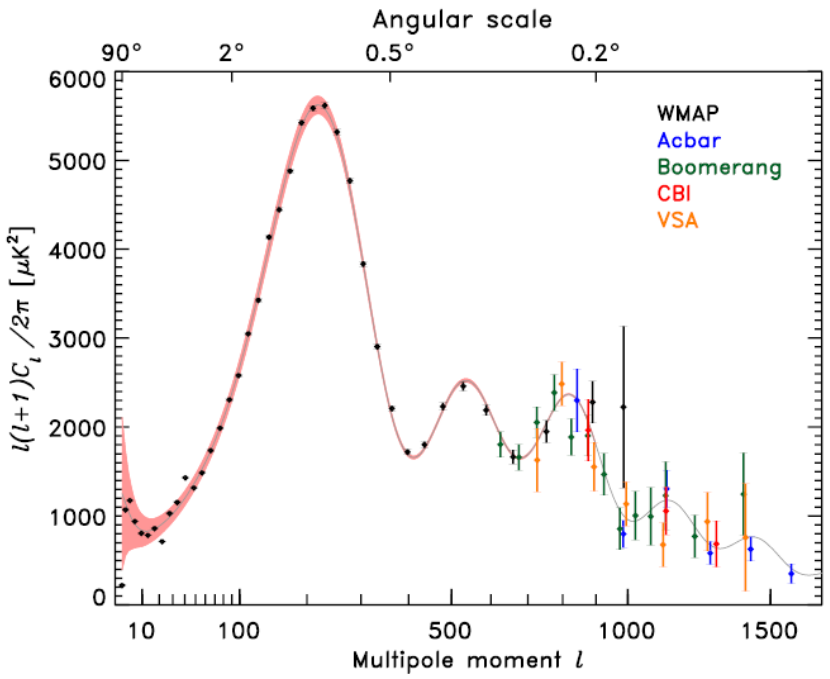




\section{Tool of Precision Cosmology}
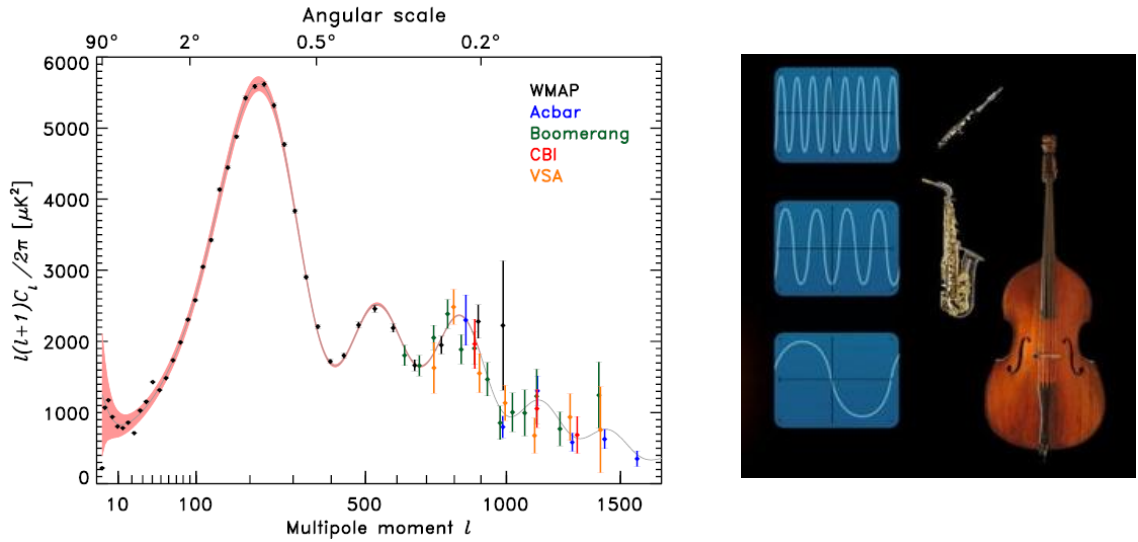

Soundscape of the sky 


\section{Tool of Precision Cosmology}
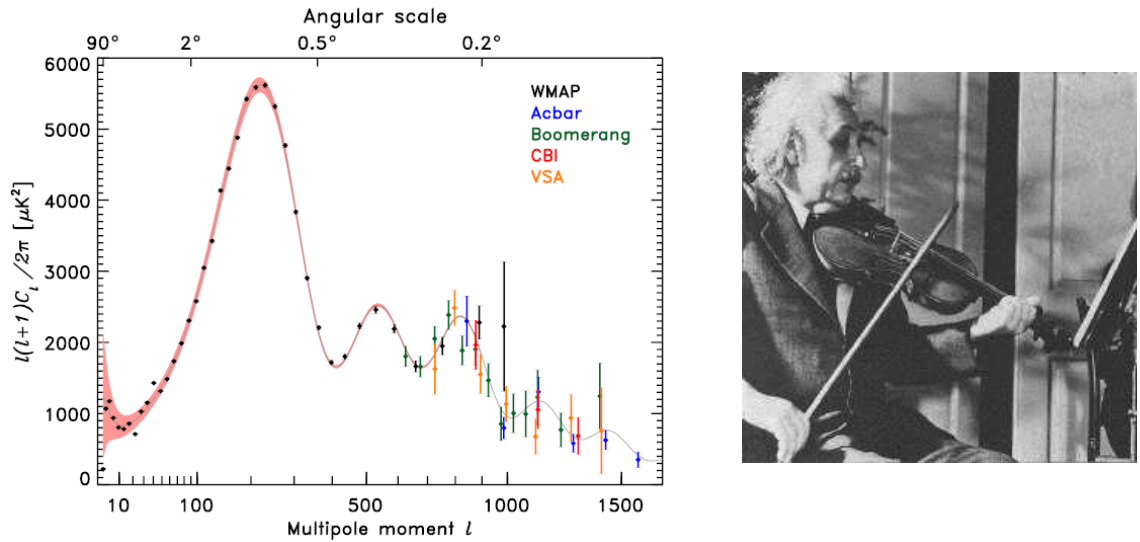

Soundscape of the sky 


\section{Tool of Precision Cosmology}
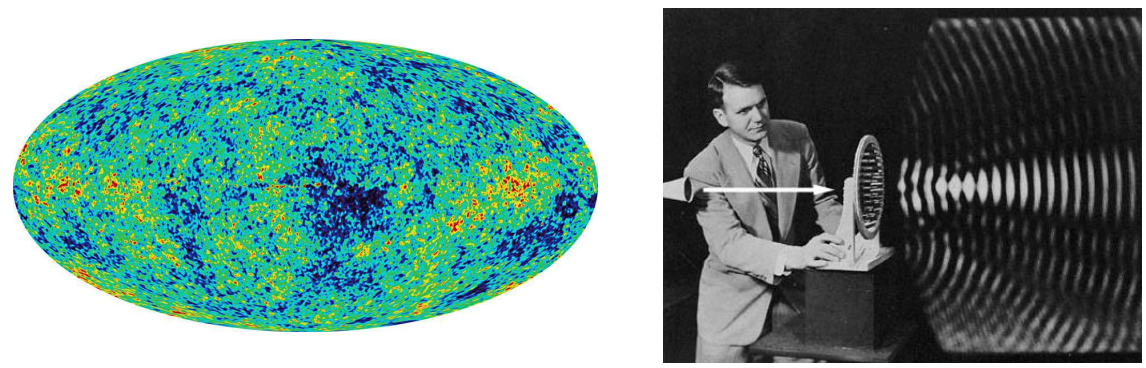

Instant photo of sound waves 


\section{Cosmological parameters}

\begin{tabular}{|c|c|c|c|c|}
\hline Description & Symbol & Value & + uncertainty & - uncertainty \\
\hline Total density & $\Omega_{\text {tot }}$ & 1.02 & 0.02 & 0.02 \\
\hline Equation of state of quintessence & $w$ & $<-0.78$ & $95 \% \mathrm{CL}$ & - \\
\hline Dark energy density & $\Omega_{\Lambda}$ & 0.73 & 0.04 & 0.04 \\
\hline Baryon density & $\Omega_{b} h^{2}$ & 0.0224 & 0.0009 & 0.0009 \\
\hline Baryon density & $\Omega_{b}$ & 0.044 & 0.004 & 0.004 \\
\hline Baryon density $\left(\mathrm{cm}^{-3}\right)$ & $n_{b}$ & $2.5 \times 10^{-7}$ & $0.1 \times 10^{-7}$ & $0.1 \times 10^{-7}$ \\
\hline Matter density & $\Omega_{m} h^{2}$ & 0.135 & 0.008 & 0.009 \\
\hline Matter density & $\Omega_{m}$ & 0.27 & 0.04 & 0.04 \\
\hline Light neutrino density & $\Omega_{\nu} h^{2}$ & $<0.0076$ & $95 \% \mathrm{CL}$ & - \\
\hline CMB temperature $(\mathrm{K})^{\mathrm{a}}$ & $T_{\text {cmb }}$ & 2.725 & 0.002 & 0.002 \\
\hline CMB photon density $\left(\mathrm{cm}^{-3}\right)^{\mathrm{b}}$ & $n_{r}$ & 410.4 & 0.9 & 0.9 \\
\hline Baryon-to-photon ratio & $\eta$ & $6.1 \times 10^{-10}$ & $0.3 \times 10^{-10}$ & $0.2 \times 10^{-10}$ \\
\hline Baryon-to-matter ratio & $\Omega_{b} \Omega_{m}^{-1}$ & 0.17 & 0.01 & 0.01 \\
\hline Fluctuation amplitude in $8 h^{-1} \mathrm{Mpc}$ spheres & $\sigma_{8}$ & 0.84 & 0.04 & 0.04 \\
\hline Low- $z$ cluster abundance scaling & $\sigma_{8} \Omega_{m}^{0.5}$ & 0.44 & 0.04 & 0.05 \\
\hline Power spectrum normalization (at $\left.k_{0}=0.05 \mathrm{Mpc}^{-1}\right)^{\mathrm{c}}$ & $A$ & 0.833 & 0.086 & 0.083 \\
\hline Scalar spectral index (at $\left.k_{0}=0.05 \mathrm{Mpc}^{-1}\right)^{\mathrm{e}}$ & $n_{s}$ & 0.93 & 0.03 & 0.03 \\
\hline Running index slope (at $k_{0}=0.05 \mathrm{Mpc}^{-1}$ ) ${ }^{e}$ & $d n_{s} / d \ln k$ & -0.031 & 0.016 & 0.018 \\
\hline Tensor-to-scalar ratio (at $k_{0}=0.002 \mathrm{Mpc}^{-1}$ ) & r & $<0.90$ & $95 \% \mathrm{CL}$ & - \\
\hline Redshift of decoupling & $z_{\text {dec }}$ & 1089 & 1 & 1 \\
\hline Thickness of decoupling (FWHM) & $\Delta z_{\text {dec }}$ & 195 & 2 & 2 \\
\hline Hubble constant & $h$ & 0.71 & 0.04 & 0.03 \\
\hline Age of universe (Gyr) & $t_{0}$ & 13.7 & 0.2 & 0.2 \\
\hline Age at decoupling (kyr) & $t_{\text {dec }}$ & 379 & 8 & 7 \\
\hline Age at reionization $(\mathrm{Myr}, 95 \% \mathrm{CL})$ ) & $t_{r}$ & 180 & 220 & 80 \\
\hline Decoupling time interval (kyr) & $\Delta t_{\text {dec }}$ & 118 & 3 & 2 \\
\hline Redshift of matter-energy equality & $z_{e q}$ & 3233 & 194 & 210 \\
\hline Reionization optical depth & $\tau$ & 0.17 & 0.04 & 0.04 \\
\hline Redshift of reionization (95\% CL) & $z_{\mathrm{r}}$ & 20 & 10 & 9 \\
\hline Sound horizon at decoupling $\left({ }^{\circ}\right)$ & $\theta_{A}$ & 0.598 & 0.002 & 0.002 \\
\hline Angular size distance to decoupling (Gpc) & $d_{A}$ & 14.0 & 0.2 & 0.3 \\
\hline Acoustic scale $^{d}$ & $\ell_{A}$ & 301 & 1 & 1 \\
\hline Sound horizon at decoupling $(\mathrm{Mpc})^{d}$ & $r_{s}$ & 147 & 2 & 2 \\
\hline
\end{tabular}




\section{Geometry of the Universe}
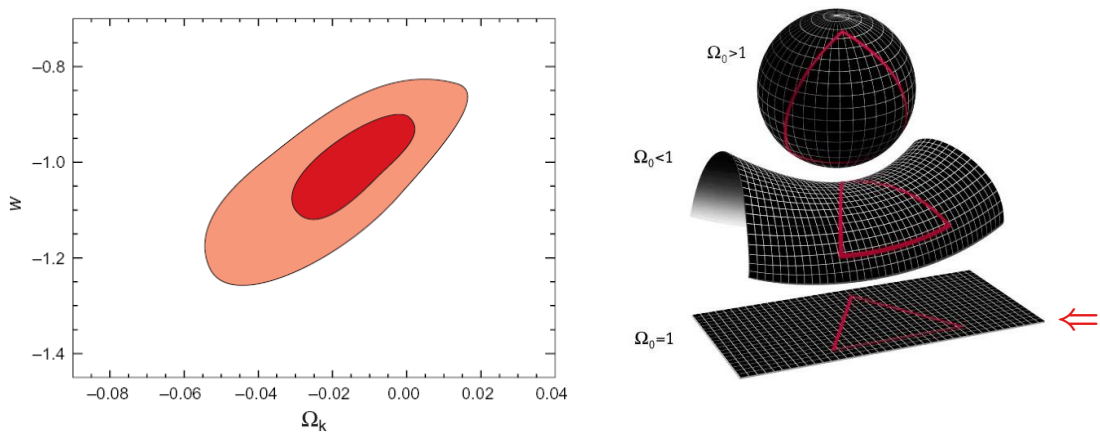

The Universe is spatially flat and is dominated by the dark energy with the equation of state $w=-1$ 


\section{Constraints on the neutrino mass}

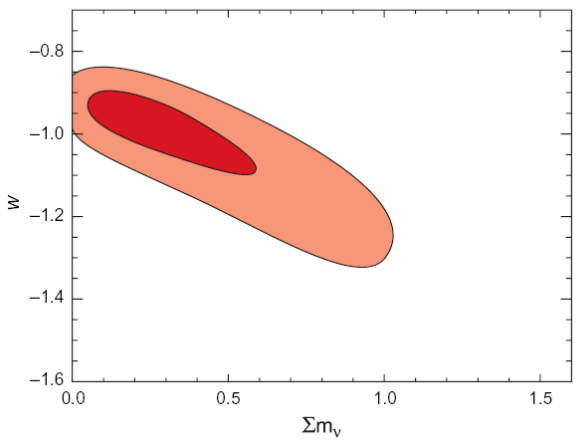

Neutrino temperature

$$
T_{\nu}=1.947 \mathrm{~K}
$$

This gives $n_{\nu i}=115 \mathrm{~cm}^{-3}$

Since $\rho_{\nu}=\sum_{i} m_{\nu i} n_{\nu i}$ we also have a constraint

$$
\Omega_{\nu}<10^{-2} \Omega_{m}
$$




\section{Constraints on the matter abundance}

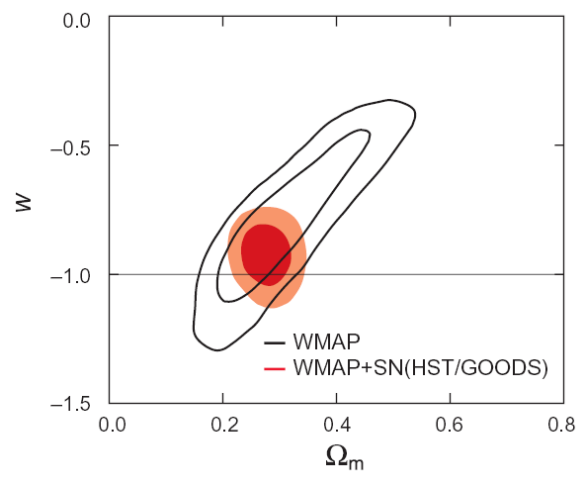




\section{Matter content in the Universe}
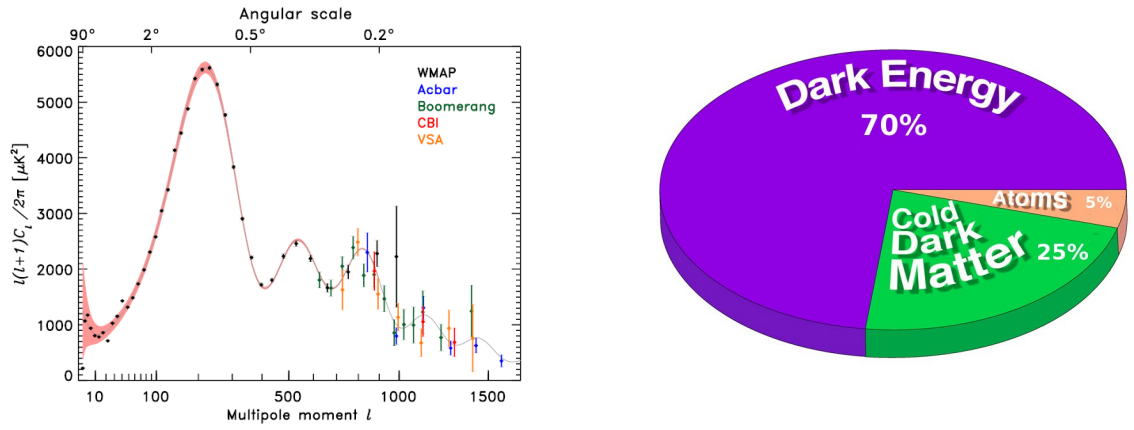

$96 \%$ of the Universe is made of unknown substance 
Missing mass is seen on all cosmological scales and reveals itself via

- Flat rotational curves in galaxies

- Gravitational potential which confines galaxies and hot gas in clusters

- Gravitational lenses in clusters

- Gravitational potential which allows structure formation from tiny primeval perturbations

- Gravitational potential which creates CMBR anisotropies

-... 


\section{Dark matter in galaxies}
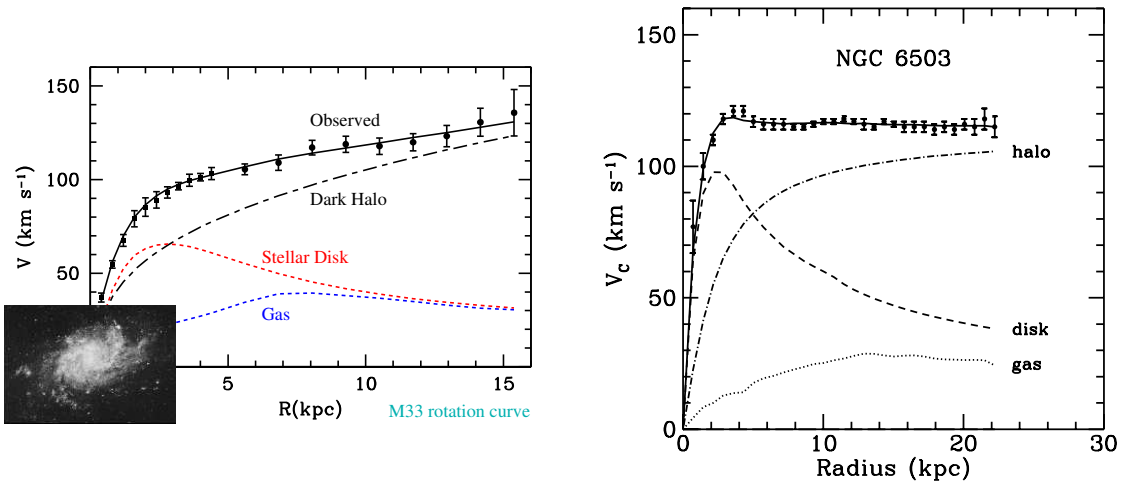

Newtonian Dynamics:

$$
\frac{v_{\mathrm{rot}}^{2}}{r}=\frac{G M(r)}{r^{2}} \rightarrow v_{\mathrm{rot}}=\sqrt{\frac{G M(r)}{r}}
$$




\section{Dark matter in clusters of galaxies}

\section{Halo structure}

- Simplest self-gravitating stationary solution which gives flat rotational curves - isothermal sphere

$$
\rho(r)=\frac{\rho_{0}}{\left(1+x^{2}\right)}, \quad \text { where } x \equiv r / r_{c}
$$

- Empirical fit to numerical simulations, Navarro, Frenk \& White (NFW) profile

$$
\rho(r)=\frac{\rho_{0}}{x(1+x)^{2}}
$$

- ... 


\section{Dark matter in clusters of galaxies}

Abell 2029

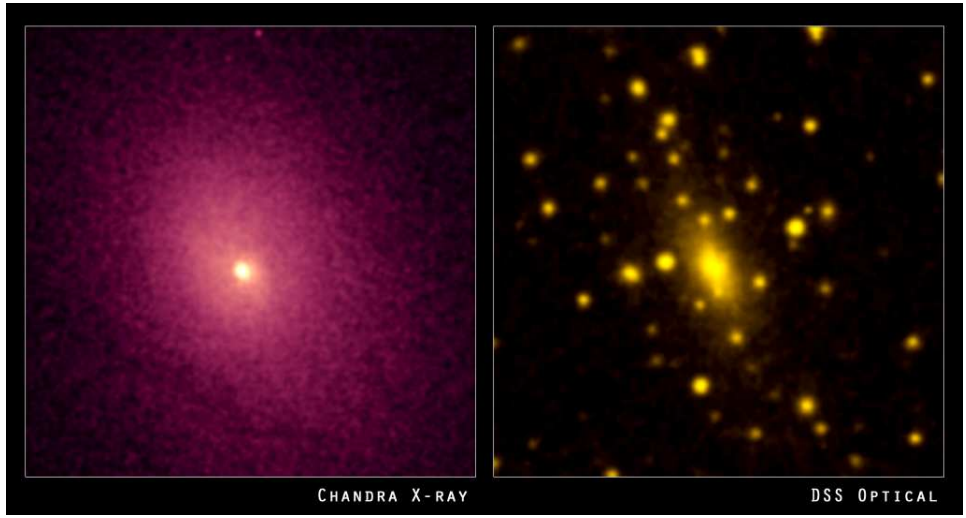

X-ray and optical data produce similar density profiles dominated by single DM component, consistent with NFW profile 


\section{Dark matter in clusters of galaxies}

Mass estimate. I.

Virial theorem $2\left\langle\boldsymbol{E}_{\text {kin }}\right\rangle+\left\langle\boldsymbol{E}_{\text {pot }}\right\rangle=0$

where $\left\langle E_{\text {kin }}\right\rangle=\frac{1}{2} N m\left\langle v^{2}\right\rangle, \quad\left\langle E_{\text {pot }}\right\rangle=-\frac{1}{2} \frac{G N^{2} m^{2}}{\langle r\rangle}$

gives for the total mass estimate, $M \equiv N m$

$$
M \sim \frac{2\langle r\rangle\left\langle v^{2}\right\rangle}{G}
$$

E.g. for Coma cluster $\frac{M}{L} \sim 300 h \frac{M_{\odot}}{L_{\odot}}$

Mass of visible galaxies $1 \%-7 \%$

Hot gas contributes $10 \%-40 \%$

The rest should be dark matter. 


\section{Dark matter in clusters of galaxies}

Mass estimate. II. Assume hot gas is in thermal equilibrium in gravitational well created by cluster. Measuring temperature profile one can reconstruct gas pressure and density and hence the gravitational potential.

Detailed modeling of Abell 2029 gives

triangles: gas contribution dot-dashed curves: stellar mass with $M / L$ of 1 and 12

solid line: NFW dark matter profile $\rho \propto \frac{1}{x\left(1+x^{2}\right)}$,

where $x \equiv r / r_{s}$ and $r_{s}=540 \mathrm{kpc}$

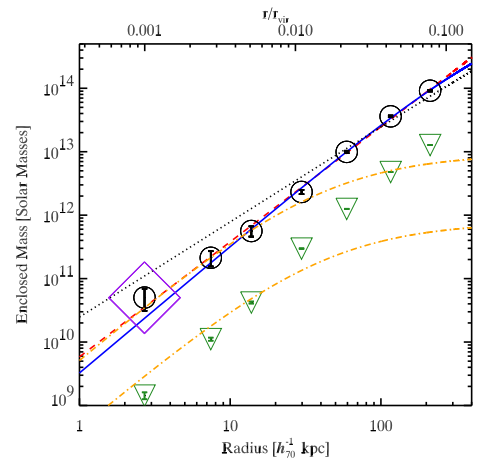

Baryonic fraction $14 \%$. This also gives $\Omega_{m} \approx 029$. 


\section{Dark matter in clusters of galaxies}

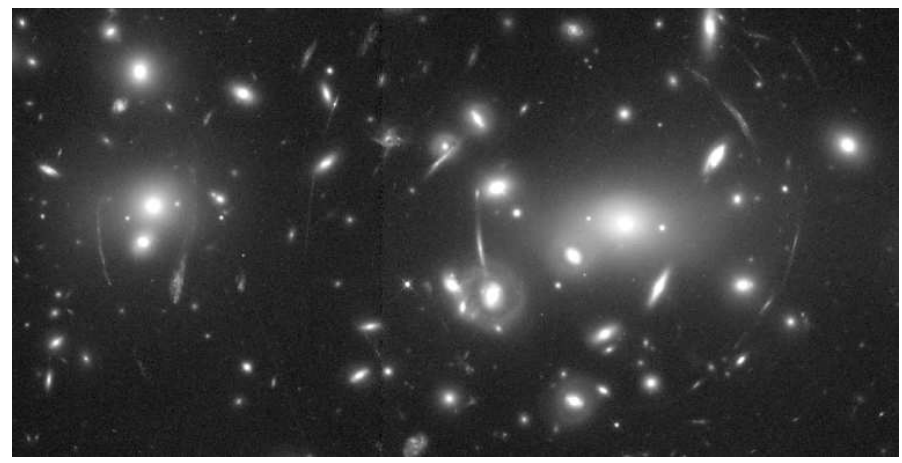

Abell 2218

Acts in this example as a gravitational lens 


\section{Structure formation and Dark Matter}

By today the structure is formed already, $\delta \rho / \rho \sim 1$. Initial perturbations were small, $\delta \rho / \rho \sim 10^{-5}$.

Perturbations do not grow in the radiation dominated epoch, during matter domination $\delta \rho / \rho \sim a$. Morover, perturbations in baryons can grow only after recombination. But

$$
\frac{a_{\text {today }}}{a_{\text {dec }}}=1+z_{\text {dec }}=1090
$$

Therefore, in a baryonic universe structure can grow only by a factor

$$
10^{3}
$$

One needs non-baryonic dark matter to facilitate structure growth 


\section{CMBR and Dark matter}
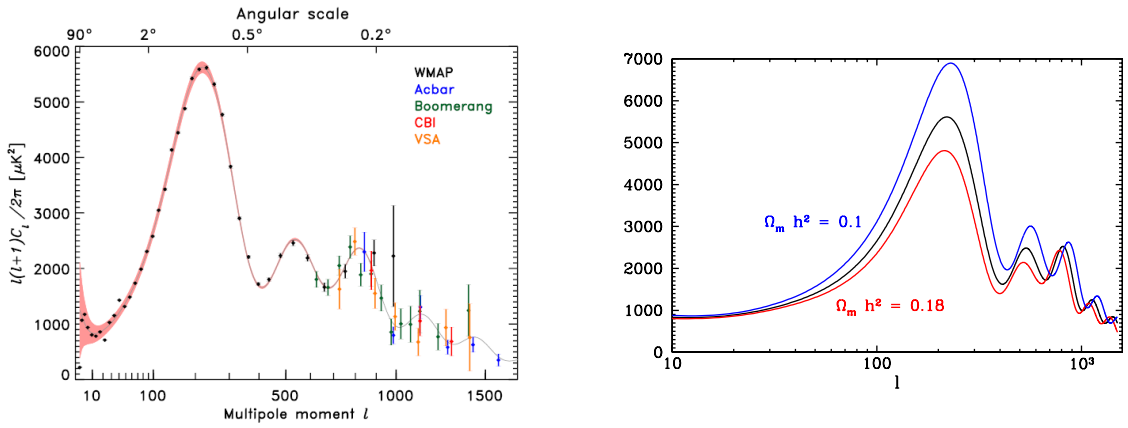

Best fit values (CMB data only): $\quad h=0.73 \pm 0.05$

$$
\Omega_{m} h^{2}=0.13 \pm 0.01, \quad \Omega_{B} h^{2}=0.023 \pm 0.001
$$

Or

$$
\Omega_{\mathrm{CDM}}=0.2 \pm 0.02
$$




\begin{tabular}{|l|l|}
\hline name & mass \\
\hline & \\
Graviton & $10^{-21} \mathrm{eV}$ \\
Axion & $10^{-5} \mathrm{eV}$ \\
Sterile Neutrino & $10 \mathrm{keV}$ \\
Mirror matter & $1 \mathrm{GeV}$ \\
WIMP & $100 \mathrm{GeV}$ \\
WIMPZILLA & $10^{13} \mathrm{GeV}$ \\
& \\
\hline
\end{tabular}




\section{Dark matter and Dark energy: what's the difference?}

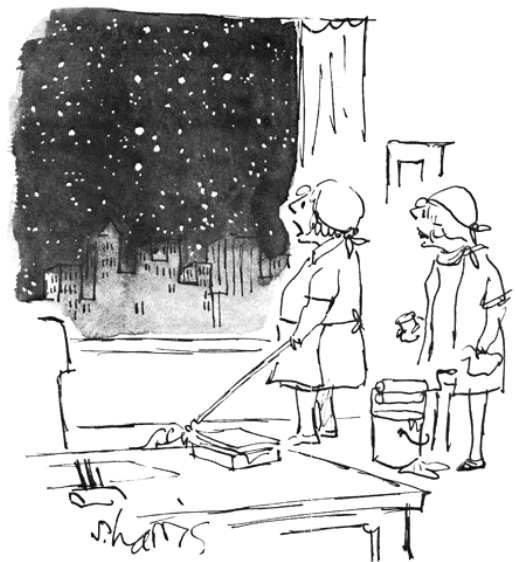

"Sure it's beautiful, but I can't help thinking about all that interstellar dust out there."
DARK MATTER:

- From the fluid dynamics point of view it behaves like a dust

- After identification of dark matter a new member in the the "zoo" of elementary particles will appear,

- and we will learn about the underlying particle physics theory, its symmetries and will single out the relevant model. 


\section{Dark matter and Dark energy: what's the difference?}

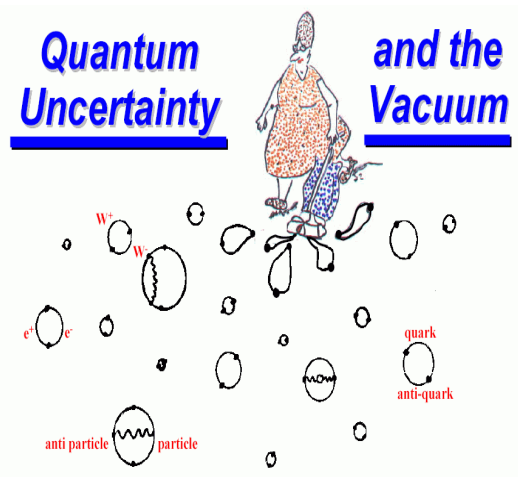

\section{DARK ENERGY:}

- Dark energy is the energy of the vacuum. It remains constant with expansion.

- We do not understand it on the fundamental level yet. (Why it is non-vanishing and at present is relevant cosmologically?)

- Understanding of it has great promise. 


\section{Evidence for Dark energy}

Age of the Universe

During mater dominated expansion $a \propto t^{2 / 3}$. Therefore

$$
H_{0} t_{0}=2 / 3
$$

Pre-WMAP measurments:

$$
H_{0}=70 \pm 7 \mathrm{~km} \mathrm{sec}^{-1} \mathrm{Mpc}^{-1}, \quad t_{0}=13 \pm 1.5 \mathrm{Gyr}
$$

and $\quad H_{0} t_{0}=0.93 \pm 0.15$

For two components, usual matter and dark energy:

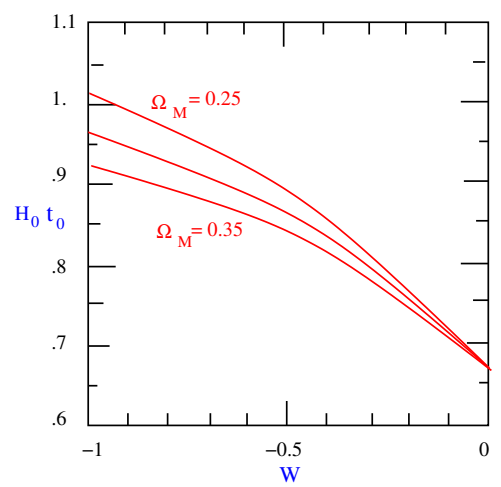


Supernovae: direct probe of the expansion history 


\section{Expansion history}

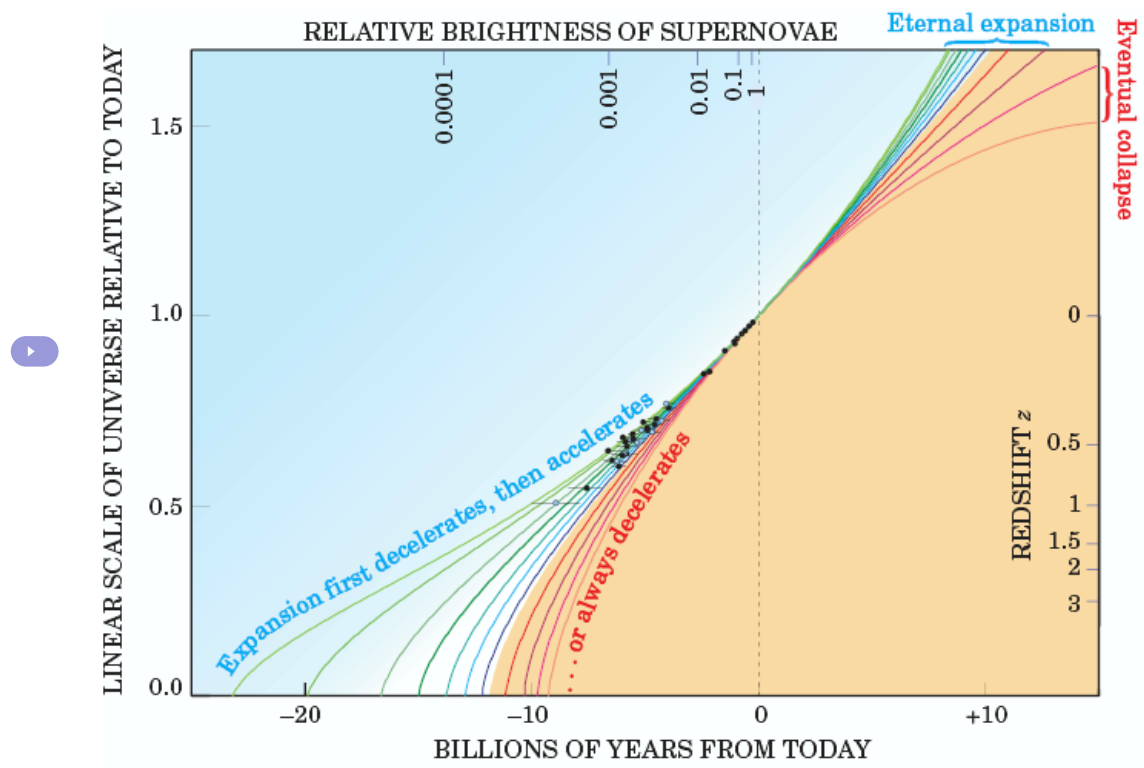




\section{Detailed Matter content}

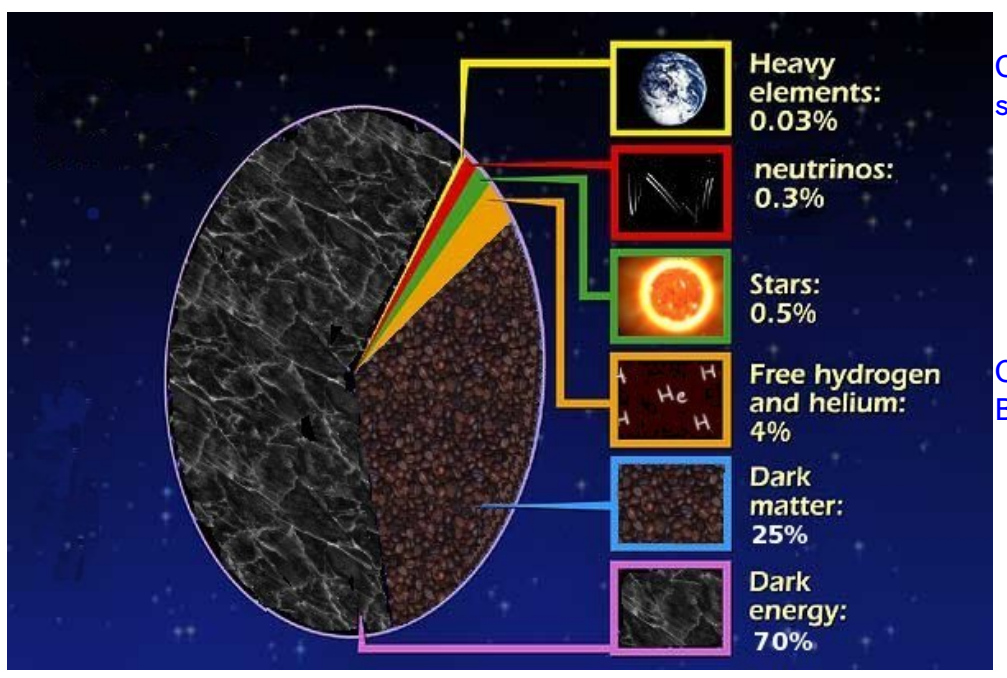

Cooked in stars

Cooked in Big Bang 


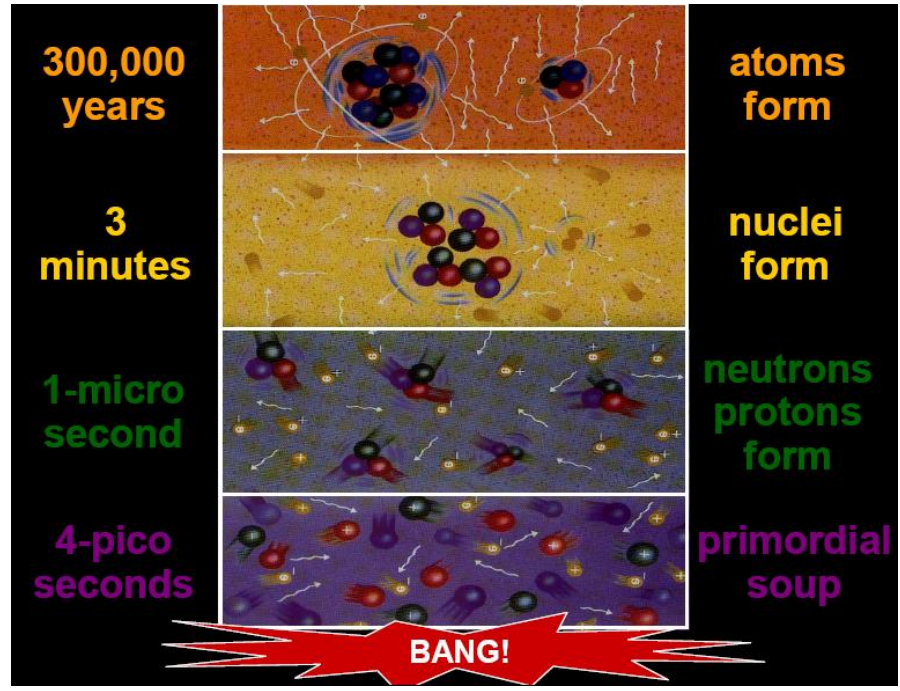




\section{Symmetry}

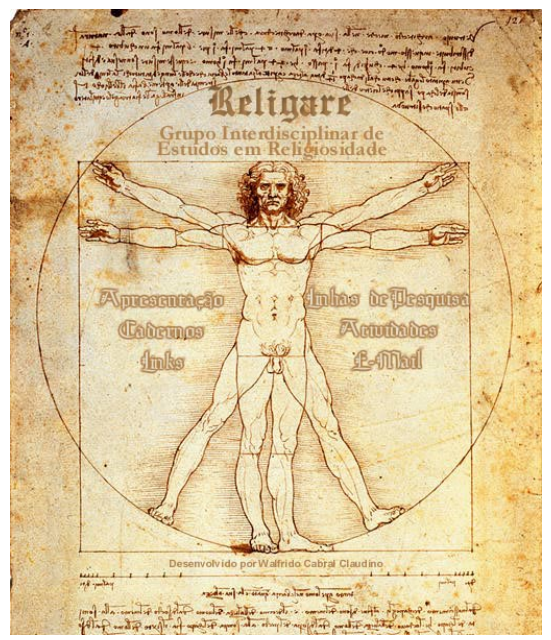

- Laws of physics do not depend upon observer or choice of coordinates

- Different interactions (or particles) can be unified in one entity

- True ultimate theory is maximally symmetric

- The symmetries are broken in our world (aka our vacuum)

Symmetry: the guiding principle of physics 


\section{Unification of forces}

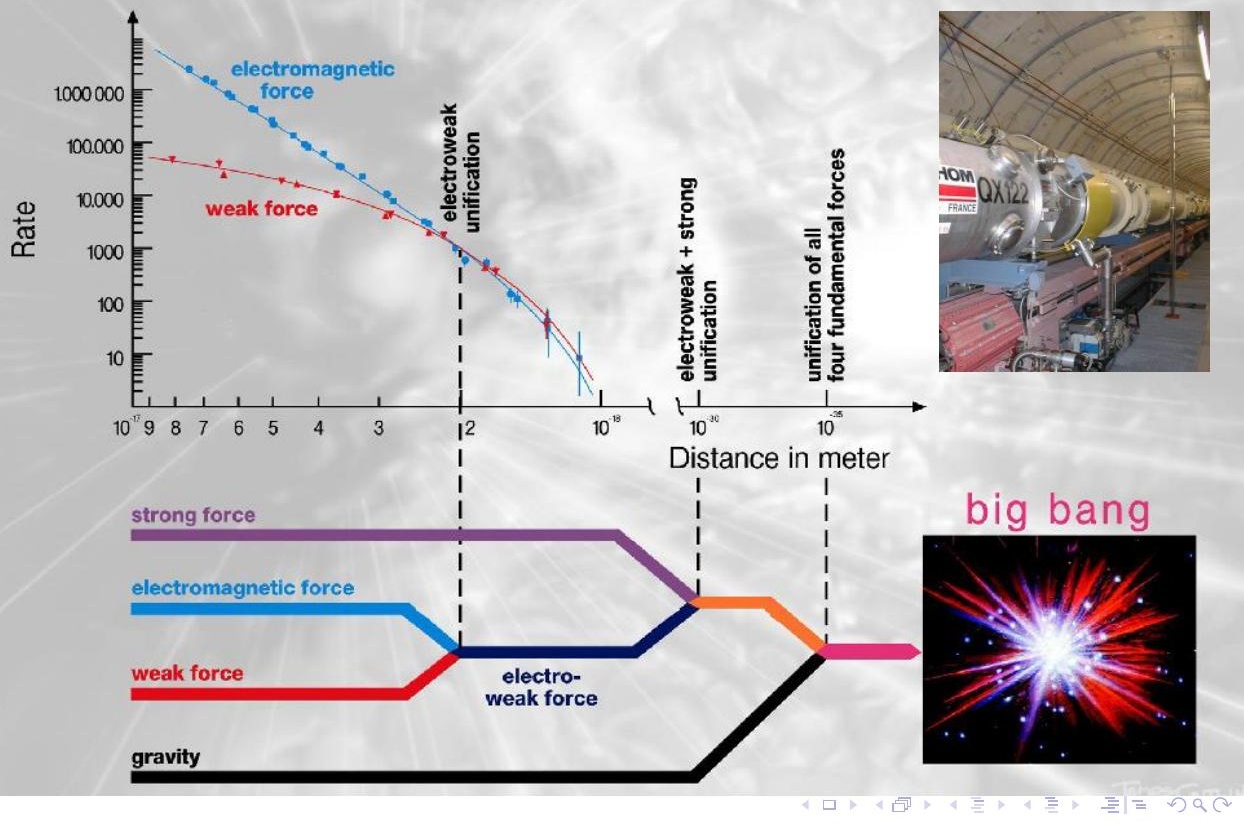




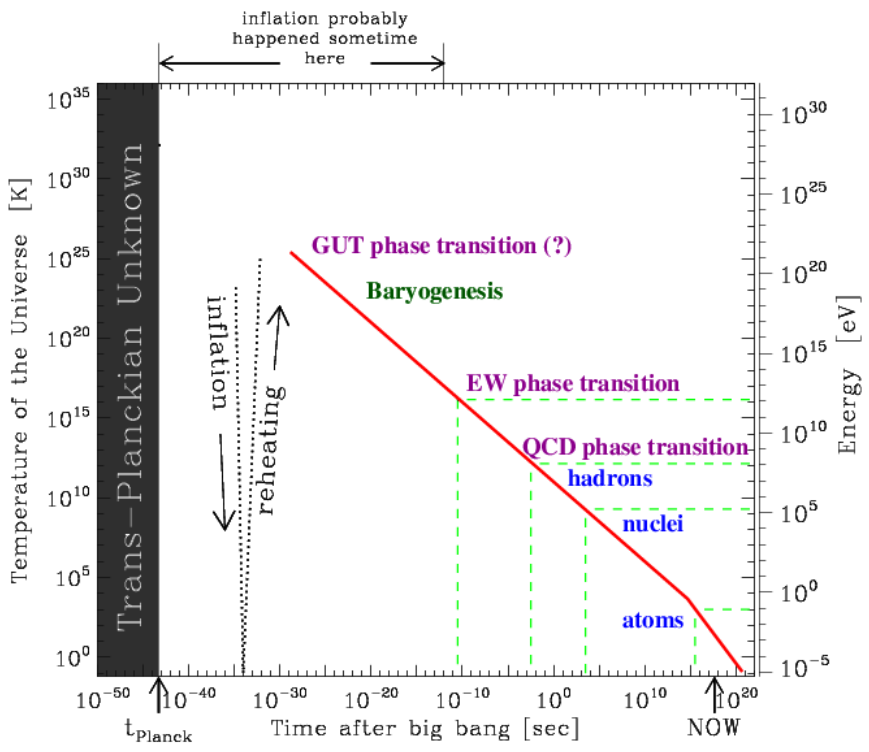


Universe is hot and expands: it was even hotter in the past.

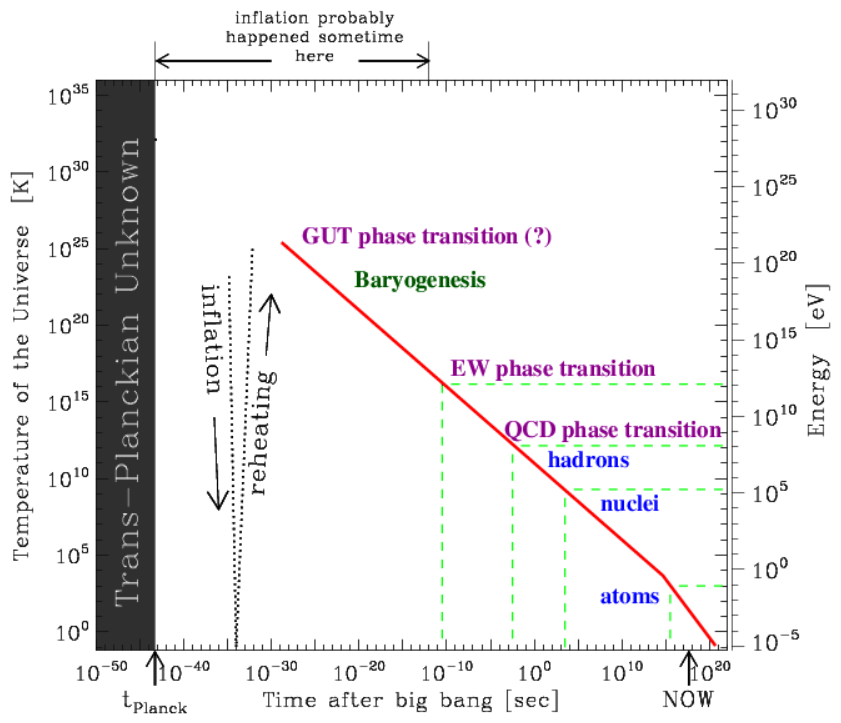




\section{Big Bang Nucleosynthesis: Helium abundace}

${ }^{4} \mathrm{He}$ is the second most abundant element, constitutes about $25 \%$. Chemical equilibrium between protons and neutrons is maintained by weak interactions

$$
n+\nu \rightleftarrows p+e^{-}, \quad n+e^{+} \rightleftarrows p+\bar{\nu},
$$

which get out of thermal equilibrium at $T_{f} \sim 1 \mathrm{MeV}$ On the other hand

$$
\Delta m \equiv m_{n}-m_{p}=1.29 \mathrm{MeV}
$$

Therefore, at freeze-out

$$
\frac{n}{p} \sim \mathrm{e}^{-\Delta m / T_{f}} \approx 0.27
$$

It is important also that neutron lifetime (980 s) is much longer than the age of the Universe at this time $(1 \mathrm{~s}) .{ }^{4} \mathrm{He}$ is the most bound among the light elements, $E_{\text {bind }} \approx 28 \mathrm{MeV}$. Therefore, almost all neutrons produced in the early universe should end up in ${ }^{4} \mathrm{He}$. 


\section{Big Bang Nucleosynthesis}

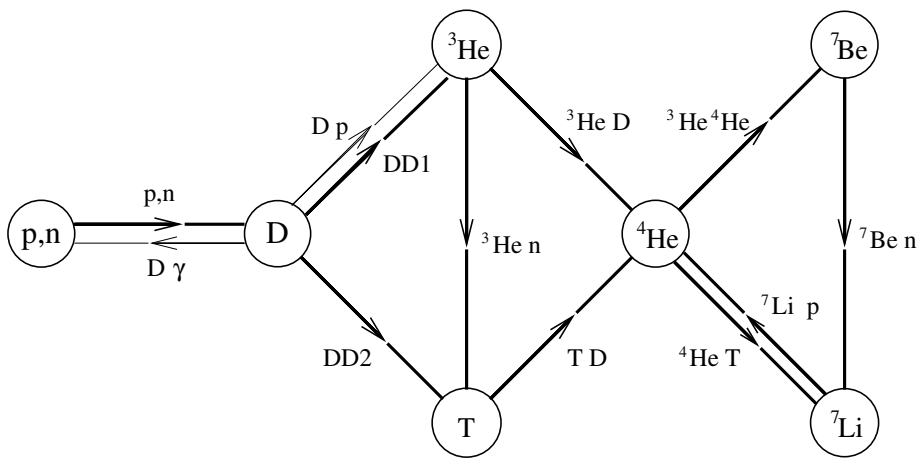

Involves numerical solution of coupled kinetic equations

$$
\frac{d n_{i}}{d t}=I\left(n_{1}, n_{2}, \ldots\right)
$$

for the concentrations of elements in the expanding Universe 


\section{BBN light element abundances}

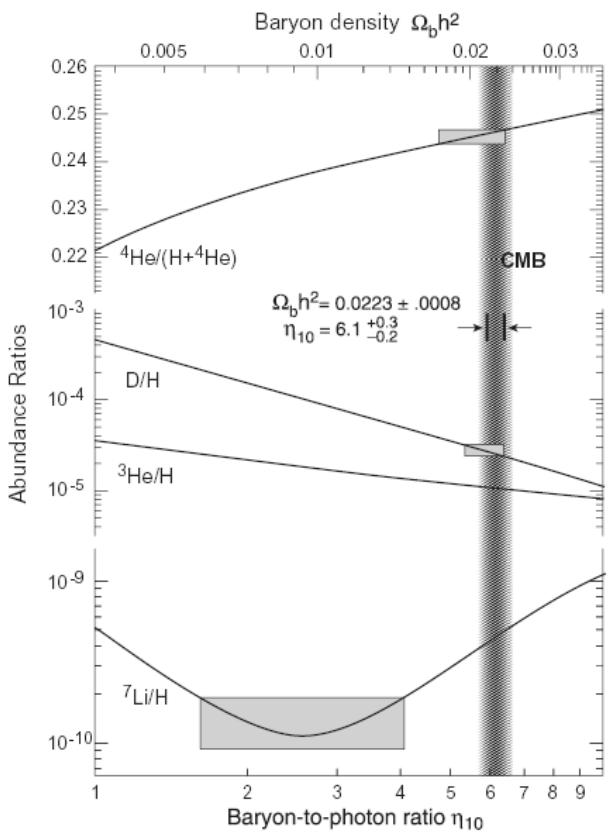

Baryon asymmetry $\eta_{10}$ is determined from CMB with a precision of $4 \%$ 


\section{Baryon asymmetry}

In a comoving volume, at late times, entropy and the number of baryons are conserved. This gives important cosmological parameter, baryon asymmetry:

$$
\eta=\frac{n_{B}}{n_{\gamma}}
$$

Observationally $\eta=(6.1 \pm 0.25) \times 10^{-10}$

This quantity should and can be understood dynamically within frameworks of the Big Bang.

Baryon asymmetry can be generated if

- Baryon number is not conserved

- C- and CP- are violated

- There are deviations from thermal equilibrium 


\section{Baryogenesis}

\section{Mechanisms}

- Grand Unified Baryogenesis

- Leptogenesis

-...
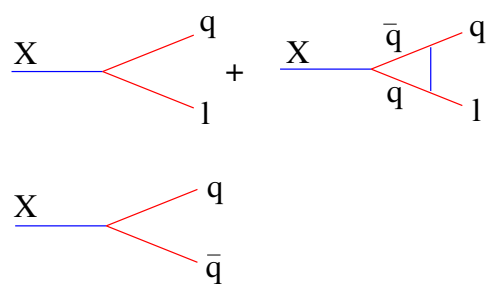

Grand Unified Baryogenesis. Out of equilibrium decays of heavy leptoquarks.

If $\mathrm{C}$ - and $\mathrm{CP}$ - are violated

$\Gamma(X \rightarrow q, l) \neq \Gamma(\bar{X} \rightarrow \bar{q}, \bar{l})$ 


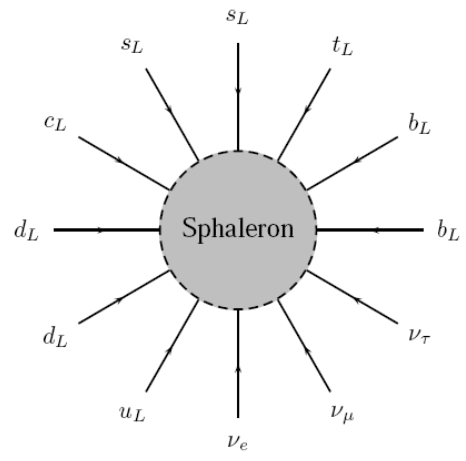

It converts three baryons to three antileptons.

At $T \gg M_{W}$ this process washes out any $B+L$

Kuzmin, Rubakov, Shaposhnikov (1985)

Leptogenesis.

Lepton asymmetry can be generated in decays or oscillations of $\nu_{R}$

Lepton asymmetry $\Rightarrow$ Sphalerons $\Rightarrow$ Baryon asymmetry 


\section{Basics of inflation}

Puzzles of classical cosmology

WHY THE UNIVERSE

is so old, big and flat?

$t>10^{10}$ years

homogeneous and isotropic? $\delta T / T \sim 10^{-5}$

- contains so much entropy? $S>10^{90}$

- does not contain unwanted relics?

(e.g. magnetic monopoles)

can be solved with hypothesis of Inflation 


\section{Basics of inflation}

\section{Definition}

"Inflation" is a period of accelerated universe expansion

$$
\ddot{a}>0
$$

Friedmann equations

$$
\ddot{a}=-\frac{4 \pi}{3} G a(\rho+3 p)
$$

We have inflation whenever $\quad p<-\rho / 3$ 


\section{Getting something for nothing}

$$
T_{\mu}^{\nu}=\left(\begin{array}{cccc}
\rho & 0 & 0 & 0 \\
0 & -p & 0 & 0 \\
0 & 0 & -p & 0 \\
0 & 0 & 0 & -p
\end{array}\right)
$$

Energy-momentum conservation $T_{; \nu}^{\mu \nu}=0$ can be written as

$$
\frac{d \rho}{d t}+3 H(\rho+p)=0
$$

Consider $T_{\mu \nu}$ for a vacuum. Vacuum has to be Lorentz invariant, hence $T_{\mu}^{\nu}=V \delta_{\mu}^{\nu}$ and we find $p=-\rho \Rightarrow \dot{\rho}=0$

Energy of the vacuum stays constant despite the expansion ! 
Consider $T_{\mu \nu}$ for a scalar field $\varphi$

$$
T_{\mu \nu}=\partial_{\mu} \varphi \partial_{\nu} \varphi-g_{\mu \nu} \mathcal{L}
$$

with the Lagrangian :

$$
\mathcal{L}=\partial_{\mu} \varphi \partial^{\mu} \varphi-V(\varphi)
$$

In a state when all derivatives of $\varphi$ are zero, the stress-energy tensor of a scalar field is that of a vacuum, $p \approx-\rho$

$$
T_{\mu \nu}=V(\varphi) g_{\mu \nu}
$$


There are two basic ways to arrange $\varphi \approx$ const and hence to imitate the vacuum-like state.

1. A. Guth: consider potential with two minima

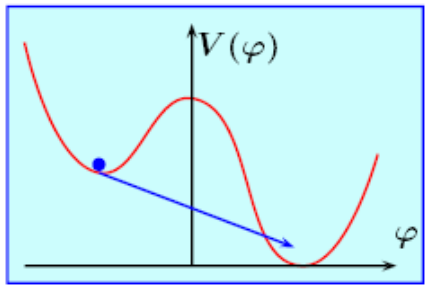

2. A. Linde: consider the simplest potential

$$
V(\varphi)=\frac{1}{2} m^{2} \varphi^{2}
$$

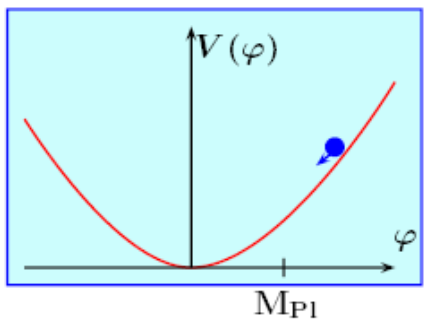


Equation of motion

$$
\ddot{\varphi}+3 H \dot{\varphi}+m^{2} \varphi=0
$$

If $\quad H \gg m$ the field rolls down slowly ("slow-roll”)

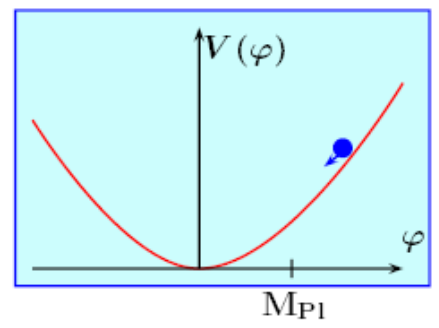

$$
H \sim m \varphi / M_{\mathrm{PI}}
$$$$
\varphi>M_{\mathrm{PI}} \quad \text { Inflation }
$$$$
\varphi<M_{\mathrm{PI}} \quad \text { Reheating }
$$ 
During Inflation the Universe is empty, in a vacuum state.

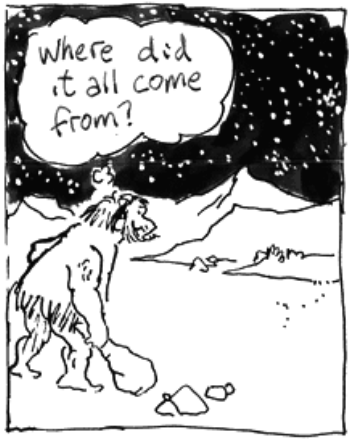

Particle physicist

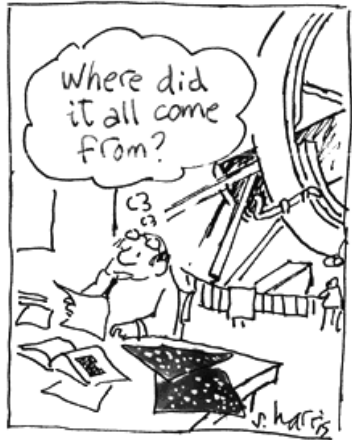

Cosmologist

Where all matter and seeds for structure formation came from ? 


\section{Predictive power of Inflation}

Fluctuations in inflaton field
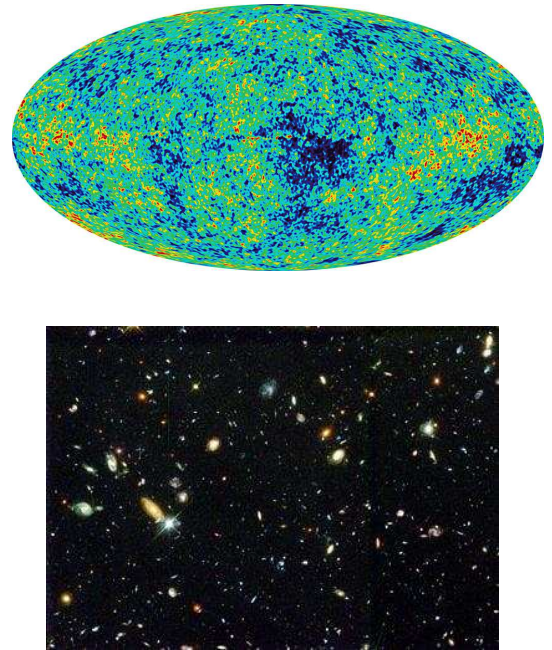

CMBR anisotropy 379,000 years after

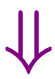

Large-scale structure 13.7 billions years after 


\section{Unified Theory of Creation}

During Inflation the Universe is "empty". But small fluctuations obey

$$
\ddot{u}_{k}+\left[k^{2}+m_{\text {eff }}^{2}(\tau)\right] u_{k}=0
$$

and it is not possible to keep fluctuations in vacuum if $m_{\mathrm{eff}}$ is time dependent 


\section{Initial linear stage}

\section{Unified Theory of Creation}

During Inflation the Universe is "empty". But small fluctuations obey

$$
\ddot{u}_{k}+\left[k^{2}+m_{\text {eff }}^{2}(\tau)\right] u_{k}=0
$$

and it is not possible to keep fluctuations in vacuum if $m_{\text {eff }}$ is time dependent

The source for $m_{\mathrm{eff}}=m_{\mathrm{eff}}(\tau)$ is time-dependence of classical backgrounds:

- Expansion of space-time, $a(\tau)$

- Evolution of the inflaton field, $\phi(\tau)$ 


$$
\ddot{u}_{k}+\left[k^{2}+m_{\text {eff }}^{2}(\tau)\right] u_{k}=0
$$

Technical remarks:

- This equation holds for all metric perturbations and for all particle species

- Equations look that simple in conformal reference frame $d s^{2}=a(\eta)^{2}\left(d \eta^{2}-d x^{2}\right)$

- For conformally coupled, but massive scalar $m_{\text {eff }}=m_{0} a(\eta)$

- $m_{\text {eff }}$ may be non-zero even for massless fields, graviton is the simplest example $m_{\text {eff }}^{2}=-\ddot{a} / a$

- Of particular interest are ripples of space-time itself

- curvature fluctuations (scalar)

- gravitons (tensor) 


$$
\ddot{u}_{k}+\left[k^{2}+m_{\mathrm{eff}}^{2}(\tau)\right] u_{k}=0
$$

Technical remarks:

- This equation holds for all metric perturbations and for all particle species

- Equations look that simple in conformal reference frame $d s^{2}=a(\eta)^{2}\left(d \eta^{2}-d x^{2}\right)$

- For conformally coupled, but massive scalar $m_{\mathrm{eff}}=m_{0} a(\eta)$

- $m_{\text {eff }}$ may be non-zero even for massless fields, graviton is the simplest example $m_{\text {eff }}^{2}=-\ddot{a} / a$

- Of particular interest are ripples of space-time itself

- curvature fluctuations (scalar)

- gravitons (tensor) 


$$
\ddot{u}_{k}+\left[k^{2}+m_{\mathrm{eff}}^{2}(\tau)\right] u_{k}=0
$$

Technical remarks:

- This equation holds for all metric perturbations and for all particle species

- Equations look that simple in conformal reference frame $d s^{2}=a(\eta)^{2}\left(d \eta^{2}-d x^{2}\right)$

- For conformally coupled, but massive scalar $m_{\text {eff }}=m_{0} a(\eta)$

- $m_{\text {eff }}$ may be non-zero even for massless fields, graviton is the simplest example $m_{\text {eff }}^{2}=-\ddot{a} / a$

- Of particular interest are ripples of space-time itself

- curvature fluctuations (scalar)

- gravitons (tensor) 


$$
\ddot{u}_{k}+\left[k^{2}+m_{\text {eff }}^{2}(\tau)\right] u_{k}=0
$$

Technical remarks:

- This equation holds for all metric perturbations and for all particle species

- Equations look that simple in conformal reference frame $d s^{2}=a(\eta)^{2}\left(d \eta^{2}-d x^{2}\right)$

- For conformally coupled, but massive scalar $m_{\mathrm{eff}}=m_{0} a(\eta)$

- $m_{\text {eff }}$ may be non-zero even for massless fields, graviton is the simplest example $m_{\text {eff }}^{2}=-\ddot{a} / a$

- Of particular interest are ripples of space-time itself

- curvature fluctuations (scalar)

- gravitons (tensor) 
Decompose a scalar field over creation and annihilation operators

$$
\phi(\mathrm{x}, t)=\frac{1}{a} \int \frac{d^{3} k}{(2 \pi)^{3 / 2}}\left[u_{k}(t) a_{\mathrm{k}} \mathrm{e}^{i \mathrm{kx}}+u_{k}^{*}(t) a_{\mathrm{k}}^{\dagger} \mathrm{e}^{-i \mathrm{kx}}\right]
$$

Regularized variance

$$
\left\langle 0\left|\varphi^{2}(x)\right| 0\right\rangle_{\text {reg }}=\frac{1}{a^{2}} \int \frac{d^{3} k}{(2 \pi)^{3}}\left(\left|u_{k}\right|^{2}-\frac{1}{2 \omega_{k}}\right) \equiv \int P_{\varphi}(k) \frac{d k}{k}
$$

Therefore, the power spectrum of field fluctuations is

$$
P_{\varphi}(k)=\frac{k^{3}}{2 \pi^{2} a^{2}}\left(\left|u_{k}\right|^{2}-\frac{1}{2 \omega_{k}}\right)
$$




\section{Inflationary perturbations}

Assume Hubble parameter during inflation is constant, $a(\eta)=-\frac{1}{H \eta}$

Mode functions of massles field $(\xi=0)$ obey

$$
\ddot{u}_{k}+\left[k^{2}-\frac{2}{\eta^{2}}\right] u_{k}=0
$$

Solution with proper initial conditions at $\eta \rightarrow-\infty$

$$
u_{k}=\frac{\mathrm{e}^{-i k \eta}}{\sqrt{2 k}}\left(1-\frac{i}{k \eta}\right)
$$

After horizon crossing $(k \eta \ll 1): \quad u_{k} \rightarrow-\frac{1}{\sqrt{2}} \frac{i}{k^{3 / 2} \eta}$ and

$$
P_{\varphi}(k) \rightarrow \frac{H^{2}}{(2 \pi)^{2}}
$$




\section{Curvature perturbations}

Spatial Curvature ${ }^{{ }^{(3)}} R \propto \frac{1}{a^{2}}$

Its perturbation $\zeta$ :

$$
\zeta=\frac{\delta a}{a}=H \delta t=H \frac{\delta \varphi}{\dot{\varphi}}
$$

Therefore

$$
P_{\zeta}(k)=\frac{H^{2}}{\dot{\varphi}^{2}} P_{\varphi}(k)
$$

and we find

$$
P_{\zeta}(k)=\frac{1}{4 \pi^{2}} \frac{H^{4}}{\dot{\varphi}^{2}}
$$




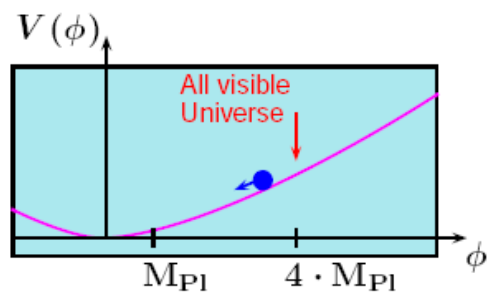

- Cosmological scales encompass small $\Delta \phi$ interval

- Potentail should be flat over this range of $\Delta \phi$

Therefore, observables essentially depend on a first few derivatives of $H$ (or $\mathrm{V}$ ) defined at some scale $\phi_{0}$. Slow-roll parameters:

$$
\begin{aligned}
& H\left(\phi_{0}\right) \\
& \epsilon \equiv \frac{M_{\mathrm{PI}}^{2}}{4 \pi}\left(\frac{H^{\prime}}{H}\right)^{2} \\
& \eta \equiv \frac{M_{\mathrm{PI}}^{2}}{4 \pi} \frac{H^{\prime \prime}}{H}
\end{aligned}
$$


In the slow-roll approximation $\ddot{\varphi}$ can be neglected and equation of moton

$$
\ddot{\varphi}+3 H \dot{\varphi}=-V^{\prime}
$$

gives

$$
\dot{\varphi}=-\frac{V^{\prime}}{3 H}
$$

Since $\rho \approx V$ we also can use

$$
H^{2}=\frac{8 \pi G}{3} V
$$

This gives for curvature perturbations

$$
P_{\zeta}(k)=\frac{H^{4}}{4 \pi^{2} \dot{\varphi}^{2}}=\frac{1}{\pi \epsilon} \frac{H^{2}}{M_{\mathrm{PI}}^{2}}
$$


Let us consider the simple model $V=\frac{1}{2} m^{2} \varphi^{2}$. We have

$$
H=\sqrt{\frac{4 \pi}{3}} \frac{m \varphi}{M_{\mathrm{Pl}}}, \quad \epsilon=\frac{M_{\mathrm{PI}}^{2}}{4 \pi \varphi^{2}}
$$

and

$$
\zeta_{k} \equiv P_{\zeta}(k)^{1 / 2}=\sqrt{\frac{16 \pi}{3}} \frac{m \varphi^{2}}{M_{\mathrm{Pl}}^{3}}
$$

Since $\delta T / T=2 \zeta_{k} / 3$ we find

$$
m \approx \frac{\delta T}{T} \frac{M_{\mathrm{PI}}}{30} \approx 10^{13} \mathrm{GeV}
$$




\section{Power spectra and consistency relation}

Power spectra of Scalar (curvature) and Tensor (gravity waves) perturbations

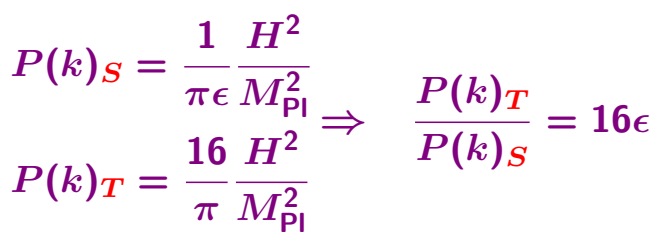

Spectra can be approximated as power law functions

$$
\begin{aligned}
& P(k)_{S}=P\left(k_{0}\right)_{S}\left(\frac{k}{k_{0}}\right)^{n-1} \\
& P(k)_{T}=P\left(k_{0}\right)_{T}\left(\frac{k}{k_{0}}\right)^{n_{T}}
\end{aligned}
$$

In slow roll parameters one finds $n-1=2 \eta-4 \epsilon, n_{T}=-2 \epsilon$ 
Reconstruction of inflaton potential, $V(\phi) \propto \phi^{n}$

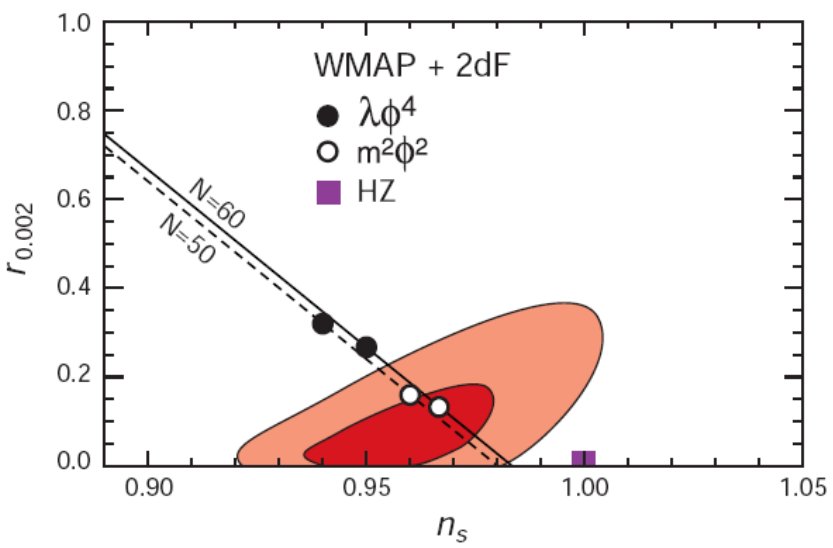

$m \sim 10^{13} \mathrm{GeV}$ 


\section{Basics of inflation}

\section{Puzzles of classical cosmology}

\section{Curvature problem and the solution}

Friedmann equation

$$
\left(\frac{\dot{a}}{a}\right)^{2}=\frac{8 \pi G}{3} \rho-\frac{k}{a^{2}}
$$

can be re-written as

$$
k=\dot{a}^{2}(\Omega-1)=\text { const }
$$

- Problem: During matter or radiation dominated stages $\dot{a}^{2}$ decreses (since $\ddot{a}<0$ ), therefore $\Omega$ is driven away from unity. To get $\Omega \sim 1$ today, one needs enormous initial fine-tuning, say at BBN epoch $\left|\Omega\left(t_{\mathrm{NS}}\right)-1\right|<10^{-15}$

- Solution: Accelerated expansion drives $\Omega$ to unity. Therefore, the problem can be solved if at early times $\ddot{a}>0$ 


\section{Basics of inflation}

\section{Puzzles of classical cosmology}

\section{Horizon problem and the solution}

Horison: $\propto t$

Physical size: $\propto a(t) \propto t^{\gamma}$

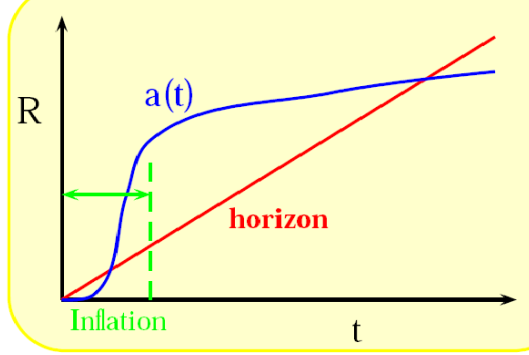

During matter or radiation dominated stages $\gamma<1$, therefore the visible universe at early times contains many causially disconnected regions.

Problem is solved if there was a period with $\gamma>1$ or $\ddot{a}>0$ 


\section{Basics of inflation}

Puzzles of classical cosmology

WHY THE UNIVERSE

is so old, big and flat?

$t>10^{10}$ years

homogeneous and isotropic? $\delta T / T \sim 10^{-5}$

- contains so much entropy? $S>10^{90}$

- does not contain unwanted relics?

(e.g. magnetic monopoles)

can be solved with hypothesis of Inflation 